\title{
Subfield-specific effects of chronic mild unpredictable stress on hippocampal astrocytes
}

\author{
Garima Virmani ｜ Priyal D'almeida ｜ Arnab Nandi | Swananda Marathe
}

Centre for Neuroscience, Indian Institute of Science, Bangalore, India

\section{Correspondence}

Swananda Marathe, Centre for

Neuroscience, Indian Institute of Science, Old TIFR Building, Gulmohar Marg, Bangalore 560012, India.

Email: swanand@iisc.ac.in; swanandlab@ gmail.com

\section{Funding information}

Department of Science and Technology, Government of India, Grant/Award Number: DST/INSPIRE/04-I/2016/000002; Science and Engineering Research Board, Grant/Award Number: ECR/2017/003240; CSIR-NET Junior and Senior Research Fellowship

\begin{abstract}
Major depressive disorder (MDD) is a debilitating neuropsychiatric illness affecting over $20 \%$ of the population worldwide. Despite its prevalence, our understanding of its pathophysiology is severely limited, thus hampering the development of novel therapeutic strategies. Recent advances have clearly established astrocytes as major players in the pathophysiology, and plausibly pathogenesis, of major depression. In particular, astrocyte density in the hippocampus is severely diminished in MDD patients and correlates strongly with the disease outcome. Moreover, astrocyte densities from different subfields of the hippocampus show varying trends in terms of their correlation to the disease outcome. Given the central role that hippocampus plays in the pathophysiology of depression and in the action of antidepressant drugs, changes in hippocampal astrocyte density and physiology may have a significant effect on behavioral symptoms of MDD. In this study, we used chronic mild unpredictable stress (CMUS) in mice, which induces a depressive-like state, and examined its effects on astrocytes from different subfields of the hippocampus. We used SOX9 and S100 $\beta$ immunostaining to estimate the number of astrocytes per square millimeter from various hippocampal subfields. Furthermore, using confocal images of fluorescently labeled glial fibrillary acidic protein (GFAP)-immunopositive hippocampal astrocytes, we quantified various morphology-related parameters and performed Sholl analysis. We found that CMUS exerts differential effects on astrocyte cell numbers, ramification, cell radius, surface area, and process width of hippocampal astrocytes from different hippocampal subfields. Taken together, our study reveals that chronic stress does not uniformly affect all hippocampal astrocytes; but exerts its effects differentially on different astrocytic subpopulations within the hippocampus.
\end{abstract}

\section{K E Y W O R D S}

astrocyte, CA1, CA3, hilus, molecular layer, Mus musculus, Sholl analysis, stress

\footnotetext{
Abbreviations: BSA, bovine serum albumin; CA1 s.r., stratum radiatum region of CA1; CA3 s.1, stratum lucidum region of CA3; CMUS, chronic mild unpredictable stress; DG, dentate gyrus; FST, forced swim test; GFAP, glial fibrillary acidic protein; MDD, major depressive disorder; OFT, open field test; PB, 0.1-M phosphate buffer; PBS, phosphate-buffered saline; PFA, paraformaldehyde; ROI, region of interest; SPT, sucrose preference test.

Edited by Ethan Hughes
} 


\section{1 | INTRODUCTION}

Major depression is associated with structural remodeling in various brain regions at a sub-cellular, cellular, and network level (Dai et al., 2019; Nestler et al., 2002). On the other hand, antidepressant therapies rely on reversing some of these alterations by inducing structural plasticity (Castrén \& Hen, 2013; Czéh et al., 2005; Santarelli et al., 2003). One of the most striking forms of structural alteration in the patients of major depressive disorder (MDD) is volumetric loss as well as hypertrophy in various regions of the limbic system. In particular, major depression induces volumetric loss in the hippocampus (Bremner et al., 2002; Brown et al., 2014; Drevets, 2000; Kempton et al., 2011; Lorenzetti et al., 2009; Nestler et al., 2002; Sheline, 2003; Sheline et al., 1996). Interestingly, the volume reduction in the hippocampus shows a strong correlation with the disease outcome. In particular, a meta-analysis from 32 different studies showed that the hippocampal volume reduction was only evident among patients who experienced multiple MDD episodes or whose illness had lasted for at least 2 years (McKinnon et al., 2009). On the other hand, patients who did not fit these criteria did not show any changes in the hippocampal volume (McKinnon et al., 2009). Furthermore, in chronic or recurrent MDD, total volume of the hippocampus showed inverse correlation with the duration of the illness (Cobb et al., 2013).

Although neuronal and spine atrophy has been thought to underlie the volume reduction (Duman \& Duman, 2015; Qiao et al., 2016; Sheline, 2003; Sousa et al., 2000), increasing evidence supports the involvement of astrocytes in the volumetric loss (Cobb et al., 2016; Czéh et al., 2005; Naskar \& Chattarji, 2019; Rajkowska et al., 1999; Rajkowska \& Stockmeier, 2013) and the pathophysiology of depression (Elsayed \& Magistretti, 2015; Marathe et al., 2018). Astrocytes are profoundly affected as a result of depression, resulting in significant atrophy (Cobb et al., 2016; Cotter et al., 2001; Miguel-Hidalgo et al., 2000, 2010; Ongür et al., 1998; Rajkowska et al., 1999; Rajkowska \& Stockmeier, 2013). Hippocampus is a relatively complex brain structure with three principal subfields, namely, dentate gyrus (DG), CA3, and CA1. In the hippocampus, the excitatory neurons are neatly arranged in thin layers of cells, such as the granule cell layer of the DG and the principal cell layers of CA3 and CA1. These neuronal layers are relatively devoid of astrocytic cell bodies. However, the regions of the hippocampus that are rich in synapses, such as the molecular layer of the DG, harbor dense populations of astrocytes. Given their location close to the synapses, any detrimental change in their morphology may have a large impact on hippocampus-dependent behaviors. It appears that the atrophy in astrocytes is not uniform and varies between different hippocampal subfields (Cobb et al., 2013, 2016; Willard et al., 2013). In particular, regionspecific changes in astrocytic density that may correlate with behavioral symptoms and the history of antidepressant use are of immense interest. Intriguingly, it was shown that in the absence of antidepressant treatment, astrocytic density in the hippocampal hilar region was reduced (Cobb et al., 2016). On the other hand, the depressed patients taking antidepressant medication did not show any such decrease in hilar astrocyte density (Cobb et al., 2016). The same study also showed an inverse correlation between area fraction of glial fibrillary acidic protein (GFAP) immunoreactivity in the CA2/3 region, and the duration of depression in suicide victims (Cobb et al., 2016). Additionally, the volume fraction of GFAP immunoreactivity was selectively decreased in the DG in females with depression, but not in males (Cobb et al., 2016). A study in depressed monkeys showed that depression was associated with reduced astrocyte density in the DG and CA1 of the anterior hippocampus (Willard et al., 2013). These results suggest that prolonged depression may exert differential effects on the astrocytes from different subfields of the hippocampal formation.

How depression may affect astrocytes from different hippocampal subfields differently is not clear. Mechanistic understanding of this phenomenon of region-specific differential effects would require this to be studied in simpler and more tractable model system. Astrocytic structural changes have been studied in animal models of depression (Czéh et al., 2005; Jang et al., 2008; Musholt et al., 2009; Naskar \& Chattarji, 2019). However, these studies have not compared the astrocytes across various hippocampal subfields along the rostro-caudal extent of the hippocampus. In this study, we sought to address whether such subfield-specific changes in astrocyte density and morphology can be found in the hippocampus of mouse model of depression as well.

In this study, we subjected C57BL/6J mice to chronic mild unpredictable stress (CMUS) that induces depressive-like state and studied the changes in the number of cells expressing astrocytic markers per square millimeter and morphology across different hippocampal subfields. We found subfieldspecific effects on cell numbers and on morphology-related parameters.

We believe that these results would prove useful to understand the mechanisms underlying diversity and differential vulnerability of hippocampal astrocytes in the pathophysiology of depression.

\section{2 | MATERIALS AND METHODS}

\section{1 | Animals}

All experiments were performed on C57BL/6J mice bred in IISc Central Animal Facility. Mice were group-housed and maintained on 12/12-hr light/dark cycle with access to food and water ad libitum. Adult male mice (25-30 g, 
4-7 months old) were used for all experiments. All procedures were carried out in accordance with the protocols approved by Institutional Animal Ethics Committee (IAEC), Indian Institute of Science. All efforts were made to reduce the number of animals by using the principle of 3R's.

\section{2 | CMUS}

Mice undergoing CMUS were transported to another room 30 min prior to the onset of stress. Control animals remained in the holding room to avoid exposure to stress-related auditory and olfactory stimuli from the CMUS-treated mice. Thirty minutes after the completion of each stressor, the mice were brought back to the holding room. One to two stressors were administered every day at random time intervals with a gap of at least $3 \mathrm{hr}$ between successive stressors (Table S1). Following stressors were used: Overcrowding (three to four mice were kept in 1-L glass beaker for $3 \mathrm{hr}$ ), cage tilt (home cages were tilted at $45^{\circ}$ for $12 \mathrm{hr}$ overnight), cold exposure (cages were kept on a layer of ice for $45 \mathrm{~min}$ ), wet bedding (2-cm-deep water was added to home cages for $3 \mathrm{hr}$ ), restraint (mice were restrained in a $50-\mathrm{ml}$ conical plastic tube with a hole in the bottom to facilitate breathing), food and water deprivation (food and water bottles were removed for $12 \mathrm{hr}$ overnight), shaker stress (cages were kept on a shaker [Tarsons Dancing Shaker MC-02 Cat no. 3080] run at $50 \mathrm{rpm}$ for $3 \mathrm{hr}$.), tone (10-kHz tone was played at about $80 \mathrm{Db}$ for $12 \mathrm{hr}$ overnight.), and white noise (white noise was played in the band of $0-20 \mathrm{kHz}$ at about $80 \mathrm{Db}$ for a period of $12 \mathrm{hr}$ overnight). Specific schedule used in this study is summarized in Table S1.

\section{3 | Forced swim test}

Forced swim test (FST) was a part of the CMUS paradigm and served the dual purpose of being one of the stressors and also as a test of behavioral analysis. Control mice underwent FST on Day 0 and Day 19. In brief, mice were allowed to swim in a 2-L glass beaker for $5 \mathrm{~min}$ and were video recorded from the side. Immobility was scored manually. Mice were deemed to be immobile when there was a lack of any discernible movement of the limbs, other than what is required to stay afloat. Time points at which mice switched from mobility to immobility and vice versa were recorded and used for further analysis. Immobility on Day 0 was compared with that on Day 19 using three parameters: total time immobile (seconds), latency to first continuous bout of immobility of $\geq 15 \mathrm{~s}$ and the duration of the longest bout of immobility (seconds). Furthermore, the change in immobility over all time points was also analyzed in the CMUS-treated mice. Tail suspension test was a part of the CMUS paradigm, but it was not used for monitoring behavioral changes since mice showed extensive tail-climbing behavior rendering the analysis inaccurate.

\subsection{Open field test}

To assess unprovoked locomotor activity, we subjected the mice to open field test (OFT) on Day 21. OFT arena consisted of a $45-\times 45-\mathrm{cm}$ square box with $35-\mathrm{cm}$-high walls under ambient lighting during the light phase. Mice were introduced in a corner and were allowed to explore the arena freely for $10 \mathrm{~min}$. Their movements were video-recorded using a topmounted webcam (Logitech C270 HD) at 15 frames per second. Mouse positions were tracked using custom-written MATLAB ${ }^{\circledR}$ programs and the total distance traveled (in centimeters) was calculated. We further computed average speed in 1-s time bins (in centimeters per second). The maximum speed was calculated as the highest average speed attained within a single 1-s bin. Maximum speed would highlight any impediment to normal locomotion in mice as a result of CMUS, while the total distance traveled would show the ability of mice to sustain the locomotor activity over the total duration of OFT and any change in exploratory behavior.

\section{5 | Sucrose preference test}

For assessing the effects of CMUS on anhedonic behavior, we subjected both the experimental groups to sucrose preference test (SPT). On Day 16, mice were habituated with two water bottles in their home cages. For the next 2 days, they were habituated with two bottles containing $2 \%$ sucrose solution in drinking water in their home cages. On Day 19, they were provided with the usual water bottle. On the testing day (Day 20), mice were singly housed at 9 a.m. and were given one bottle each of drinking water and $2 \%$ sucrose solution for $12 \mathrm{hr}$. The positions of the bottles were swapped every $3 \mathrm{hr}$ to eliminate a possible side bias. At the end of the testing period, the volume of water and sucrose consumed was calculated. Sucrose preference index was calculated as (volume of sucrose - volume of water)/(volume of sucrose + volume of water). In the CMUS group, mice were subjected to stressors as per the schedule during SPT habituation.

\section{6 | Tissue preparation}

Twenty-four hours following the last stressor, mice were anaesthetized using isoflurane and were sacrificed by transcardial perfusion with phosphate-buffered saline followed by $4 \%$ paraformaldehyde. Serial floating coronal sections (40 $\mu$ m thick) were cut using a cryostat (Leica, CM 1850) and 
were stored at $-20^{\circ} \mathrm{C}$ in a freezing mixture $(2: 1: 1$ mixture of 0.1-M phosphate buffer [PB]: ethylene glycol: glycerol) until further use.

\subsection{Immunohistochemistry and imaging}

Immunohistochemistry protocol was based on those described earlier (Marathe et al., 2015; Yanpallewar et al., 2010). In brief, the sections were removed from the freezing mixture and washed three times with PB. Sections were then blocked for $1 \mathrm{hr}$ with $10 \%$ normal donkey serum $+3 \%$ bovine serum albumin (BSA) $+0.3 \%$ TritonX100 in $0.1-\mathrm{M}$ PB. Sections were incubated overnight at $4^{\circ} \mathrm{C}$ with primary antibodies (Goat pAb anti-SOX9, 1:200; R\&D systems, AF3075; chicken pAb anti-GFAP, 1:1,000, Novus, NBP1-05198 and rabbit pAb anti-S100 $\beta, 1: 1,000$, Synaptic systems, 287003). On the second day, the sections were washed three times with PB and incubated for $2 \mathrm{hr}$ at room temperature with secondary antibodies (donkey anti goat 568, abcam, ab175474; goat anti chicken Alexa Flour 594, abcam, ab150172; and donkey anti rabbit Alexa Flour 488, abcam, ab150073). After incubation, sections were washed three times in PB and were mounted on a glass slide in the mounting medium with DAPI (Abcam ab104139). The slides were imaged on a Zeiss LSM 880 airyscan confocal microscope with a Plan-Apochromat 20X/0.8 M27 objective. 1024X1024 pixels, 8-bit images were acquired at $0.860-\mu \mathrm{m}$ z-step size.

\subsection{Analysis of cell numbers per square millimeter}

For calculating the number of astrocytes per square millimeter, three sections per mouse were used per data point. Three to four mice were used per group. z-stacks from the confocal images of SOX9 and S100 $\beta$-positive cells were flattened with maximum intensity projection. A region of interest (ROI) was selected encompassing the given hippocampal subfield. We restricted our ROIs in CA3 largely to stratum lucidum and in CA1 to stratum radiatum. We calculated the number of SOX9 and S100ß-positive nuclei and divided it by the area of the selected ROI, using ImageJ. The numbers were then converted to the number of astrocytes per square millimeter by multiplying by an appropriate factor $\left(1 \mathrm{pixel}=0.48 \mu \mathrm{m}^{2}=0.48 / 1,000,000 \mathrm{~m}\right.$ $\mathrm{m}^{2}$ ). In order to estimate the astrocyte density across the rostro-caudal axis, the data were generated from sections from different bregma levels. The sections between bregma levels -1.46 to -1.94 were labeled as rostral, -2.06 to -2.46 as intermediate, and sections beyond -2.54 were labeled as caudal.

\section{9 | Morphological analysis}

Morphological analysis was done using Ghosh Lab Sholl analysis plugin in ImageJ. In brief, maximum intensity projection of confocal images containing GFAP-stained astrocytes was generated using ZEN black edition 3.0 SR and saved in TIFF format. The 2-D images were then binarized in ImageJ. We then used "analyze particles" tool to eliminate the background using size exclusion. Single cells were cropped out and surface area was measured by pixel count. Next, we filled holes in the thresholded image using "fill holes" tool in ImageJ. Following this step, the cells were skeletonized using skeletonize plugin in ImageJ to generate cells with single pixel-wide processes. The pixel count of the skeletonized cell was calculated to estimate the total process length. Surface area of every cell was divided by its total process length to generate the average process width for that cell. The centroid for Sholl analysis was selected based on the morphology of the original GFAP-stained images. Sholl analysis plugin from Ghosh lab was then used to generate Sholl analysis vectors. A 5-period moving average was calculated for every cell to generate smooth curves for Sholl analysis, as polynomial regressions were deemed unsuitable for our datasets. Approximately 60-150 cells (from four sections per mouse) from three to four mice per group were used for Sholl analysis per region per group. The results were represented as mean $\pm S E M$ of all cells. Area under the Sholl curve for every cell was calculated by taking the sum of the Sholl vector. Enclosing radius of each cell was calculated by the length of the respective Sholl vector after removing the zeros.

\subsection{0 | Statistical analysis}

All statistical analyses were performed using GraphPad Prism 8.0.2. Results are expressed as mean \pm SEM for Sholl analysis, FST, SPT, and OFT and as box and whiskers plots for two group comparisons of cell densities and morphological parameters. Boxes represent the 25th-75th percentile, whereas whiskers represent the minimum and maximum value in the corresponding dataset, with median represented as a horizontal line across the box and mean as "+." Requisite sample sizes were determined using power analysis. Comparison of behavioral parameters between Day 0 and the last time point was done using paired $t$ test. Analysis of FST behavior over several timepoints was performed using multiple comparisons with one-way analysis of variance (ANOVA) followed by Dunnett's multiple comparisons test. OFT, SPT, and cell count comparisons between two groups were made using unpaired $t$ test. The $F$ values for unpaired $t$ tests with non-nested designs were reported as $F$ (DFn and DFd). The analysis of astrocyte 
(a)

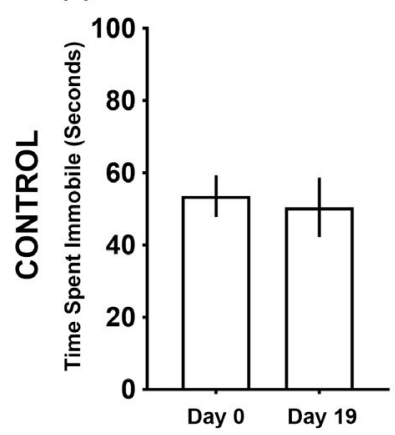

(d)

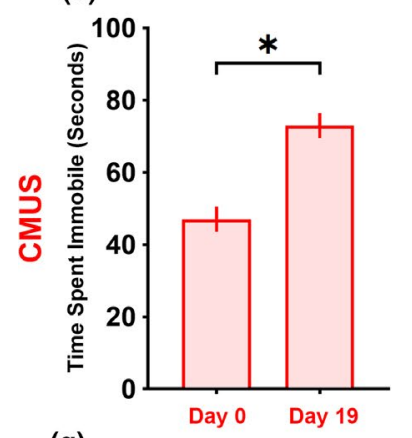

(g)

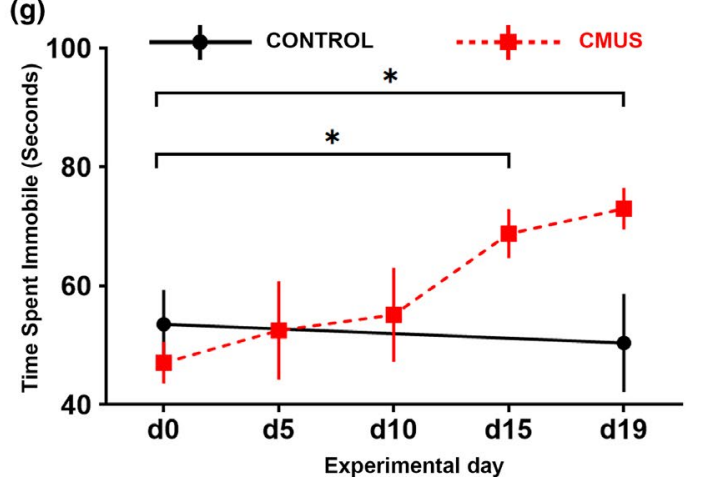

(b)

(e)
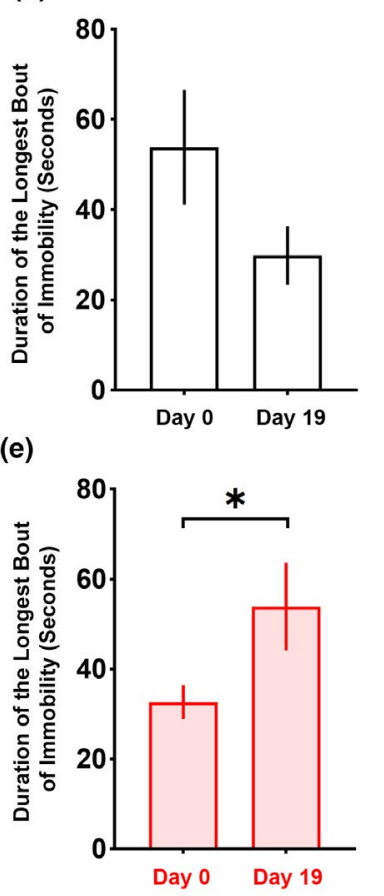

(h)

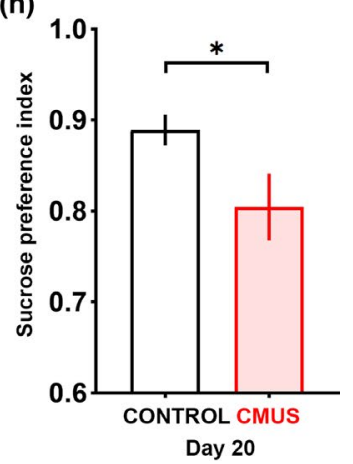

FI G URE 1 Effects of chronic mild unpredictable stress (CMUS) on depressive-like behavior. Mice were subjected to 21 days of CMUS, while the controls were handled similarly without exposure to stress. The depressive-like behavior was monitored using forced swim test (FST) and sucrose preference test (SPT). The control mice did not show any change in the time spent immobile in FST between Day 0 and Day 19 (a). The control mice also did not show any change in the duration of the longest bout of immobility and the latency to a continuous bout of immobility of $15 \mathrm{~s}$ or longer $(\mathrm{b}, \mathrm{c})$. On the other hand, the CMUS-treated mice showed a significant increase in time spent immobile from Day 0 to Day 19 (d). The CMUS-treated mice also showed a significant increase in the duration of the longest bout of immobility (e) and a decrease in the latency to the first continuous bout of immobility of $15 \mathrm{~s}$ or longer (f). The line graph shows a gradual increase in the time spent immobile in FST in the CMUS-treated mice, with statistically significant increase seen at Day 15 and Day 19 of the experiment (g). Control mice underwent FST only on Day 0 and Day 19 to avoid FST-induced stress. The controls did not show a statistically significant difference between the two timepoints (g). Mice were also subjected to SPT on Day 20 of the experiment to test for anhedonia. CMUS-treated mice showed a significant decrease in the sucrose preference index as compared to the control mice (h). $n=6-8$ mice per group. Data represented as mean $\pm S E M$. ${ }^{*} p<0.05$. All comparisons from (a) to (f) were made using paired Student's $t$ test. Analysis in (g) was performed using one-way analysis of variance followed by Dunnett's multiple comparison test. SPT analysis in (h) was performed using unpaired Student's $t$ test

numbers per square millimeter along the rostro-caudal axis was done using mixed effects model to account for the interactions between the bregma levels and stress exposure. This was followed by corrected Sidak's post hoc test. For two group comparisons of morphology-related parameters, normality of the datasets was determined using Kolmogorov-Smirnov normality test with DallalWilkinson-Lilliefor approximation. The distribution was deemed to be normal (Gaussian) at the alpha of 0.05. Two group comparisons were made using nested $t$ test to determine statistical significance. Nested $t$ test was used to account for the nested design in experimental samples, where analyzed cells came from different biological replicates (mouse identity). For Sholl analysis, the data were analyzed using two-way repeated measures ANOVA with Geisser-Greenhouse correction to account for possible 
unequal variances (Wilson et al., 2017). This was followed by corrected Sidak's post hoc test.

\section{3 | RESULTS}

\subsection{Effects of CMUS on depressive-like behavior}

To study the effects of stress on astrocyte density and morphology, we subjected mice to 21 days of CMUS as described in Section 2 (Table S1). The emergence of depressive-like symptoms was confirmed using FST and SPT (Figure 1). In FST, the CMUS-treated mice showed a statistically significant increase in time spent immobile from Day 0 to Day 19 (Day 0:47.04 \pm 3.51 , Day 19:72.96 \pm 3.49$)(p=0.004$, paired Student's $t$ test) (Figure 1d), while the control mice did not show any change (Day 0:53.54 \pm 5.77 , Day 19:50.38 \pm 8.25 ) ( $p=0.72$, paired Student's $t$ test) (Figure 1a). The CMUStreated mice also showed an increase in the duration of the longest bout of immobility (Day 0:32.63 \pm 3.79 , Day 19:53.88 \pm 9.74) $(p=0.03$, paired Student's $t$ test $)$ (Figure 1e) and a decrease in the latency to a continuous bout of immobility of $15 \mathrm{~s}$ or longer (Day 0:134.3 \pm 26.18 , Day 19:57.75 \pm 17.73$)(p=0.04$, paired Student's $t$ test $)$ (Figure 1f). On the other hand, the control mice did not show any change in these two parameters (duration of the longest bout: Day 0:53.83 \pm 12.74 , Day 19:29.83 $\pm 6.51 ; p=0.17$, paired Student's $t$ test) (latency to first immobility bout of $\geq 15$ s: Day 0:115.0 \pm 9.35, Day 19:144.8 $\pm 45.99 ; p=0.60$, paired Student's $t$ test) (Figure 1b,c).

Furthermore, we also analyzed the FST behavior over the duration of stress in the CMUS-treated mice. FST was done as a part of CMUS paradigm, and it served as an additional stressor as well. We found a gradual increase in immobility over time with statistically significant increase seen at Day 15 (adjusted $p=0.04$, one-way ANOVA, and Dunnett's multiple comparisons test) and Day 19 (adjusted $p=0.01$, one-way ANOVA, and Dunnett's multiple comparisons test) as compared to Day 0 (Figure $1 \mathrm{~g}$ ). Increased immobility in FST as a result of CMUS could arise from decreased locomotor behavior and thus may not reflect increased depression-related despair. Hence, to rule out the possibility of locomotor deficits affecting FST results, we analyzed the unprovoked motor activity in mice using OFT on Day 21 (Figure S1a). We found a significant increase in the distance traveled in CMUS-treated mice as compared to controls (Control: 2,432 \pm 243.3, CMUS: 3,215 \pm 213.7) $(p=0.03$, unpaired Student's $t$ test, $F(7,5)=1.028)$ (Figure 1b). We also analyzed the maximum average speed achieved in a 1-s time bin. We found no difference between the two experimental groups in maximum speed (control: $190.4 \pm 10.93$, CMUS: $218.2 \pm 23.89)(p=0.36$, unpaired Student's $t$ test, $F(7,5)=6.369$ ) (Figure S1c). Increased locomotor activity, as shown by the increase in the distance traveled in OFT as a result of chronic stress has been shown earlier (Grønli et al., 2005; Sequeira-Cordero et al., 2019). Increased in locomotion may counteract the CMUS-mediated decrease in mobility. Hence, increased immobility in FST in CMUS-treated mice is likely a result of stress-induced despair.

We also analyzed anhedonia behavior in the control and CMUS mice on Day 20 of the experiment using SPT. We saw a significant decrease in the sucrose preference index in CMUS-treated mice as compared to the controls (Control: $0.89 \pm 0.02$, CMUS: $0.80 \pm 0.04)(p=0.05$, unpaired Student's $t$ test, $F(4,5)=3.95$ ) (Figure 1h). Taken together, these results show that the 21-day CMUS paradigm used in this study induced depressive-like behavior in the experimental mice. (a)

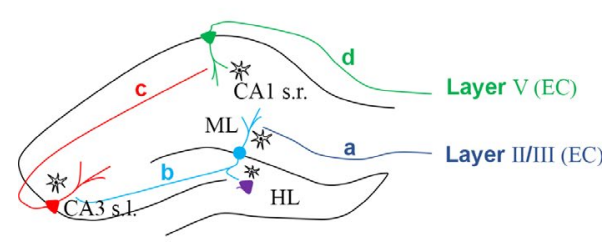

(b)

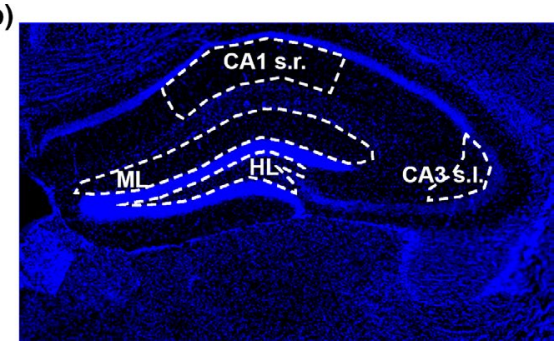

F I G U RE 2 Hippocampal circuitry and region of interest (ROI) selection. (a) Hippocampus is characterized by trisynaptic loop comprising of (a) inputs through perforant pathway originating from Layers II and III of the entorhinal cortex (EC), forming synapses with dentate gyrus (DG) granule cells in the molecular layer (ML). (b) The mossy fiber pathway originating from the DG granule cells and forming synapses with mossy cells in the hilus (HL) as well as pyramidal neurons of the CA3 in the CA3 stratum lucidum (CA3 s.l.). (c) The schaffer collaterals originating from CA3 pyramidal neurons and forming synapses with the CA1 pyramidal neurons in the stratum radiatum of the CA1 (CA1 s.r.). (d) Output from the CA1 pyramidal neurons goes back to the Layer V of the EC. (b) Image of the entire hippocampus captured through a $4 \times$ objective showing DAPIpositive nuclei. The ROIs used throughout this study have been outlined with white dotted lines. The ROIs were located in regions rich in synapses and devoid of the principal excitatory neurons of the hippocampus 


\section{2 | Effects of CMUS on SOX9 and S100ק- positive cell numbers per square millimeter across hippocampal subfields}

We used SOX9 to count the number of astrocytes per square millimeter across different hippocampal subfields (Figure 2). SOX9 was recently shown to be a specific nuclear marker to label adult astrocytes (Sun et al., 2017). In addition to SOX9, we also used $\mathrm{S} 100 \beta$ as another astrocytic marker. In all the hippocampal subfields that we analyzed from C57BL/6J mice, we found a near complete overlap between cells expressing S100 $\beta$ and GFAP (Figure S2). GFAP was used to analyze astrocyte morphology later in this study.

In order to assess the effects of CMUS on the astrocyte numbers, we estimated the number of astrocytes per square millimeter as described in Section 2. We found a statistically significant decrease in SOX9-immunopositive cell density in the molecular layer of the DG (Control: $613.9 \pm 15.18$,
CMUS: $524.2 \pm 14.57)(p<0.0001$, unpaired Student's $t$ test, $F(47,35)=1.447)$ (Figure 3a). SOX9-positive density analysis in the hilar region of the DG also showed a significant decrease in the CMUS-treated mice (control: 1,292 \pm 82.90 , CMUS: $808.6 \pm 82.13)(p=0.0002$, unpaired Student's $t$ test, $F(23,17)=1.358)($ Figure $3 b)$. In stratum lucidum region of CA3, we found a significant decline in the SOX9-expressing cell density in CMUS-treated mice compared to controls (control: $459.0 \pm 17.40$, CMUS: $373.6 \pm 20.55)(p=0.003$, unpaired Student's $t$ test, $F(23,16)=1.012)($ Figure 3c). We then quantified the SOX9-positive cell density in the stratum radiatum region of CA1. However, we did not find a statistically significant difference in the number of SOX9-expressing cells per square millimeter in CA1 (control: $414.4 \pm 16.26$, CMUS: $399.1 \pm 17.41)(p=0.53$, unpaired Student's $t$ test, $F(23,17)=1.163)($ Figure $3 d)$.

We next quantified the density of $S 100 \beta$ expressing cells in different hippocampal subfields. We did not find a difference (a)

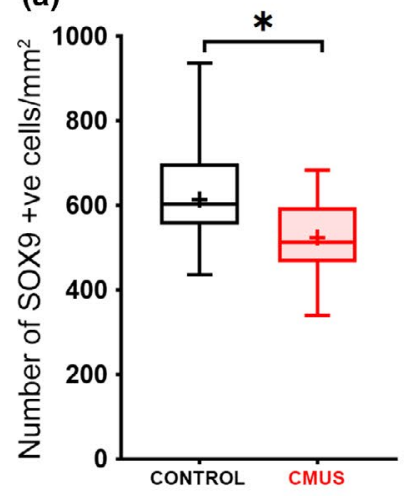

(e)

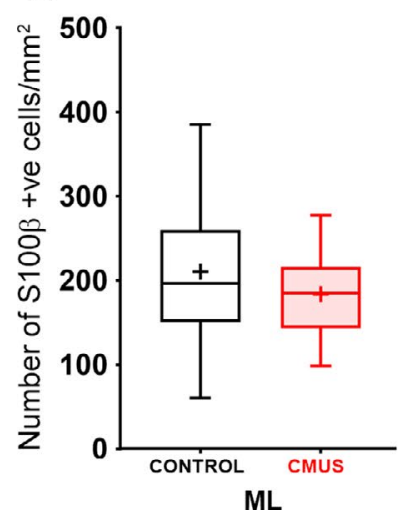

(b)

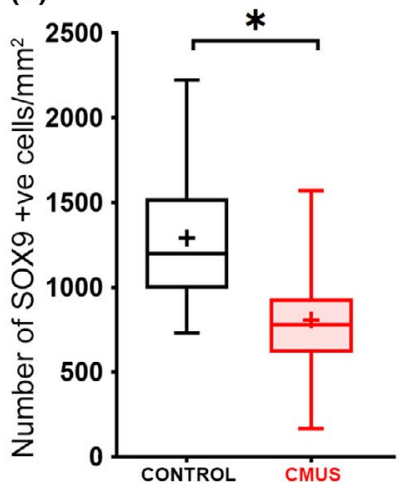

(f)

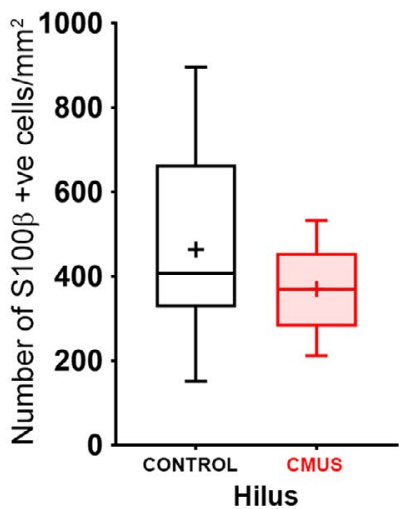

(c)

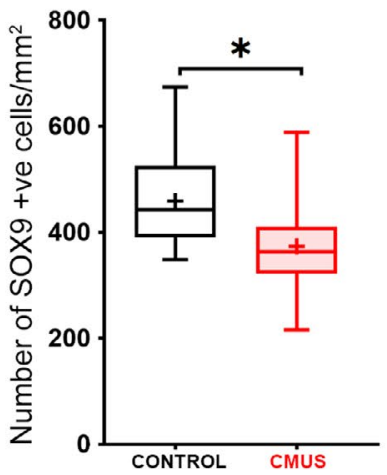

(g)

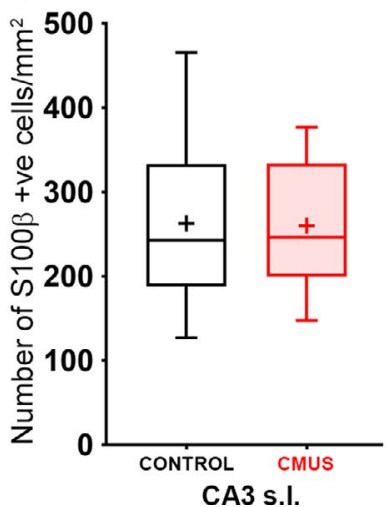

(d)

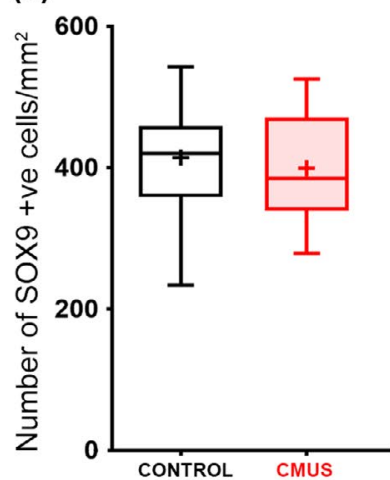

(h)

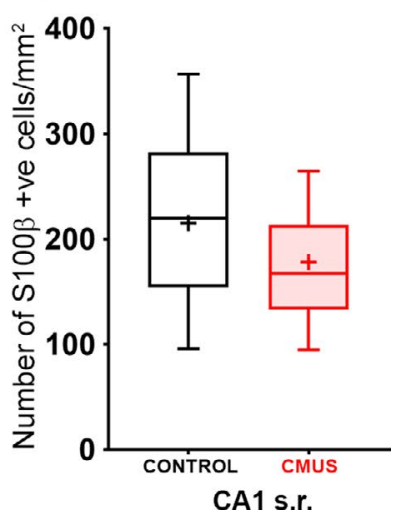

F I G U RE 3 Effects of chronic mild unpredictable stress (CMUS) on SOX9 and S100 $\beta$-positive cell numbers per square millimeter in different hippocampal subfields. Mice were subjected to 21 days of CMUS, while the controls were handled similarly without exposure to stress. SOX 9 and $\mathrm{S} 100 \beta$ immunostained astrocytes were imaged on a confocal microscope. Region of interest (ROI) was marked on 2-D maximum intensity projections encompassing the desired subfield and the number of cells counted was divided by the area of the ROI in square millimeters. The data were represented and analyzed as the number of cells per square millimeter. We observed a statistically significant decrease in the density of SOX9expressing cells in the molecular layer of the DG (a), hilus (b) and the stratum lucidum region of CA3 (c); while no differences were observed in the stratum radiatum region of CA1 (d). We did not find statistically significant differences in the molecular layer of the DG (E), hilus (f), stratum lucidum of the CA3 (g), or stratum radiatum of the CA1 (h) in the density of S100 $\beta$ expressing cells. $n=3-4$ mice per group. Data represented as box and whisker's plot. ${ }^{*} p<0.05$. All comparisons made using unpaired Student's $t$ test 
in S100ß-positive cell density in the molecular layer of the DG (control: $210.3 \pm 12.22$, CMUS: $183.7 \pm 8.599)(p=0.096$, unpaired Student's $t$ test, $F(40,31)=2.588)$ (Figure 3e). S100 $\beta$ positive cell density analysis in the hilar region of the DG revealed no significant difference between the groups in the hilus (Control: $463.3 \pm 44.45$, CMUS: $369.5 \pm 26.85)(p=0.11$, unpaired Student's $t$ test, $F(20,14)=3.836$ ) (Figure 3f). Overall, the astrocyte numbers in the hilus, as counted using SOX9 and S100 $\beta$, was approximately twice that in the molecular layer, which was consistent with previous studies (Figure 3a,b,e,f). In stratum lucidum region of CA3, we did not find any difference between CMUS-treated mice and control mice (control: $262.9 \pm 20.73$, CMUS: $260.1 \pm 18.42)(p=0.923$, unpaired Student's $t$ test, $F(21,15)=1.740$ ) (Figure $3 \mathrm{~g}$ ). We then quantified the S100ß-immunopositive cell density in the stratum radiatum region of CA1. Again, we did not find a statistically significant difference in the number of astrocytes per square millimeter in CA1 (control: $215.3 \pm 16.57$, CMUS: $178.2 \pm 12.26)(p=0.09$, unpaired Student's $t$ test, $F(19$, $16)=2.150)($ Figure $3 h)$.

Taken together, these results indicate that CMUS causes a significant decrease in SOX9-expressing cells per square millimeter in the molecular layer of the DG, hilus, and the stratum lucidum of CA3 but do not induce a statistically significant change in the number of S100ß-positive cells per square millimeter in any of the hippocampal subfields that we tested when sections are taken spanning the entire rostrocaudal extent.

\section{3 | Effects of CMUS on SOX9 and S100ק- positive cell numbers per square millimeter in rostral, intermediate, and caudal regions of the hippocampus}

Next, we sought to address whether the cell density is differentially affected between the rostral, intermediate and the caudal regions of different hippocampal subfields. We took sections from rostral (Bregma level -1.46 to -1.94 ), intermediate (Bregma level -2.06 to -2.46 ) and caudal (Bregma level -2.54 to -2.92 ) to estimate the cell density.

In the molecular layer of the DG, we did not find any difference between the two groups in rostral (control: $585.4 \pm 22.81$, CMUS: $533.5 \pm 20.00)(p=0.27)$, and the intermediate (control: $603.3 \pm 18.20$, CMUS: $536.9 \pm 30.40$ ) $(p=0.22)$ sections (Figure 4e). However, we saw a statistically significant decrease in the density of SOX9-expressing cells in the caudal sections (control: $650.60 \pm 34.70$, CMUS: $502.90 \pm 27.99)(p=0.008)$ (mixed effects analysis to assess interactions between rostro-caudal level and stress treatment, followed by corrected Sidak's post hoc test) (Figure 4e). In the rostral region, we saw a significant decrease in the SOX9-positive cell density (control: 1,157 \pm 156.00 , CMUS:
$599 \pm 84.25)(p=0.034)$, whereas there was no significant difference in the astrocyte density in the intermediate (control: $1,566 \pm 137.1$, CMUS: $881.9 \pm 200)(p=0.07)$ and the caudal (control: $1,102 \pm 76.24$, CMUS: $992.1 \pm 119.7)(p=0.84)$ hilar sections (mixed effects analysis to assess interactions between rostro-caudal level and stress treatment, followed by corrected Sidak's post hoc test) (Figure 4a,b,f). Furthermore, we assessed if CMUS affects the SOX9-immunopositive cell density in the stratum lucidum of the CA3 along the rostrocaudal axis. However, we did not find any statistically significant difference in the SOX9-immunopositive cell density in rostral (control: $516.9 \pm 34.19$, CMUS: $380.7 \pm 44.19$ ) $(p=0.09)$, intermediate (control: $436.8 \pm 23.47$, CMUS: $365.0 \pm 34.86)(p=0.34)$ and caudal (control: $433.3 \pm 28.42$, CMUS: $372.3 \pm 19.71)(p=0.28)$ sections of CA3 (mixed effects analysis to assess interactions between rostro-caudal level and stress treatment, followed by corrected Sidak's post hoc test) (Figure $4 \mathrm{~g}$ ). In the stratum radiatum region of the CA1, we also found no differences in the rostral (control: $476.9 \pm 21.91$, CMUS: $423.7 \pm 30.26)(p=0.45)$, intermediate (control: $378.1 \pm 22.60$, CMUS: $391.5 \pm 29.14)(p=0.98)$ and caudal (control: $400.6 \pm 29.34$, CMUS: $376.7 \pm 31.49$ ) $(p=0.93)$ sections (mixed effects analysis to assess interactions between rostro-caudal level and stress treatment, followed by corrected Sidak's post hoc test) (Figure 4h).

In the molecular layer of the DG, we did not find any difference between the two groups in S100 $\beta$-immunopositive cell density in rostral (control: $217.2 \pm 19.26$, CMUS: $179 \pm 12.2)(p=0.29)$, intermediate (control: $174 \pm 19.82$, CMUS: $178.8 \pm 16.14)(p=0.99)$, or caudal (control: $229.3 \pm 21.73$, CMUS: $194.2 \pm 17.85)(p=0.53)($ mixed effects analysis to assess interactions between rostro-caudal level and stress treatment, followed by corrected Sidak's post hoc test) (Figure 4i). In the rostral region of the hilus, we saw a significant decrease in the S100 $\beta$-immunopositive cell density (control: $709.3 \pm 36.99$, CMUS: $414.3 \pm 38.9$ ) $(p=0.0006)$, whereas there was no significant difference in the S100ß-immunopositive cell density in the intermediate (control: $258.9 \pm 39.17$, CMUS: $287.2 \pm 32.46)(p=0.93)$, and the caudal (control: $401.2 \pm 19.36$, CMUS: $405.3 \pm 53.15$ ) ( $p=0.99$ ) hilus (mixed effects analysis to assess interactions between rostro-caudal level and stress treatment, followed by corrected Sidak's post hoc test) (Figure 4c,d,j). Furthermore, we assessed if CMUS affects the S100 $\beta$-immunopositive cell density in the stratum lucidum of the CA3 along the rostrocaudal axis. However, we did not find any statistically significant difference in the $\mathrm{S} 100 \beta$-immunopositive cell density in rostral (control: $355.8 \pm 31.67$, CMUS: $300.5 \pm 30.3$ ) $(p=0.54)$, intermediate (control: $186.9 \pm 16.73$, CMUS: $228.8 \pm 17.17)(p=0.30$ ), and caudal (control: $248 \pm 27.34$, CMUS: $242.7 \pm 39.89)(p=0.99)$ regions of CA3 (mixed effects analysis to assess interactions between rostro-caudal level and stress treatment, followed by corrected Sidak's post 
(a)

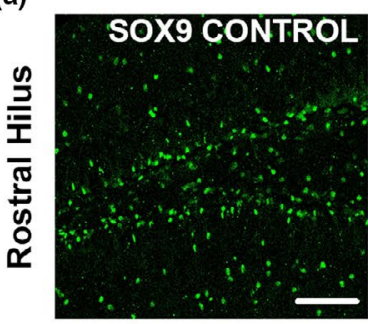

(e)

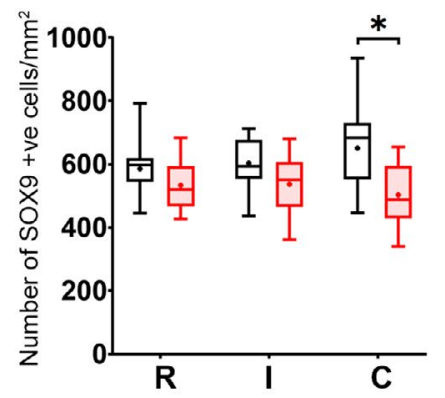

(i)

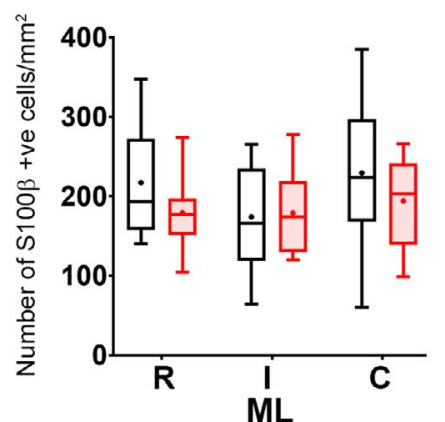

(f)

(j) (b)

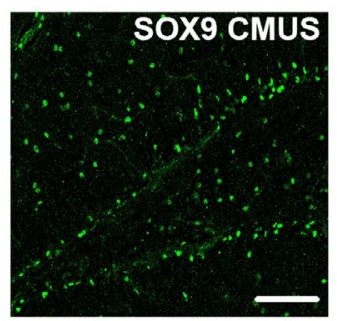

(c)

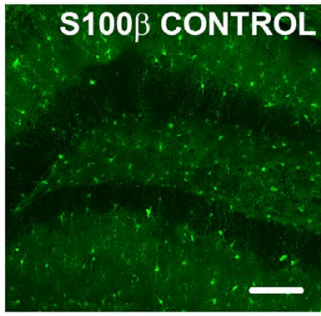

(d)
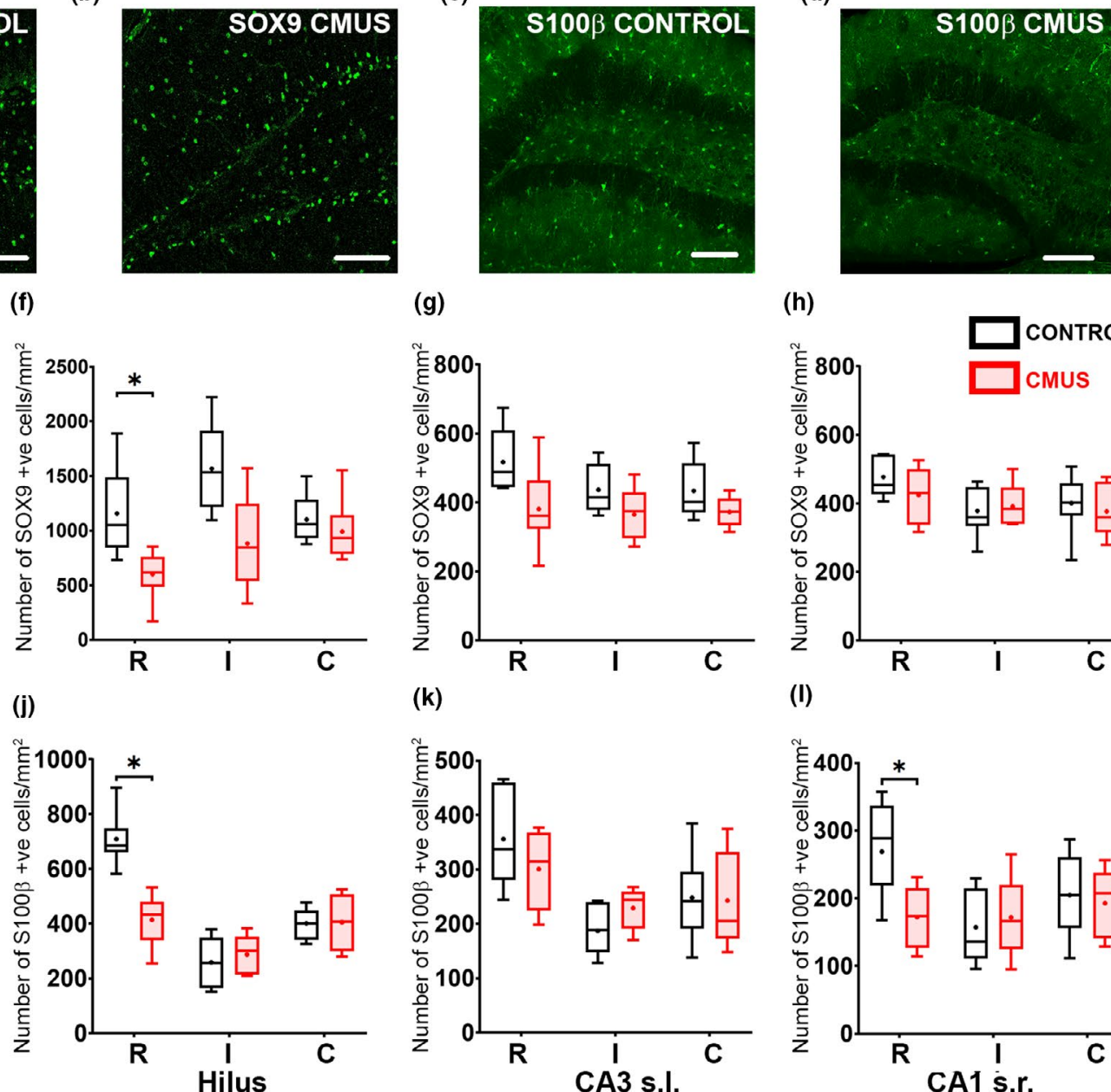

(g)

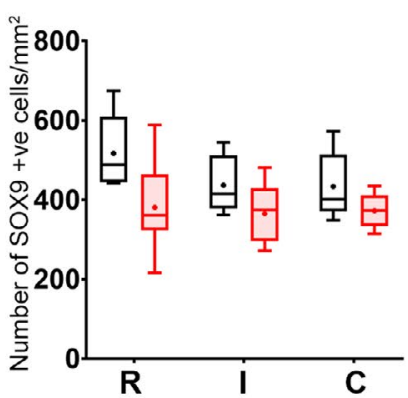

(k)

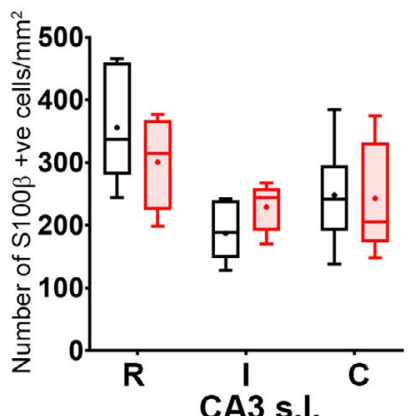

(h)

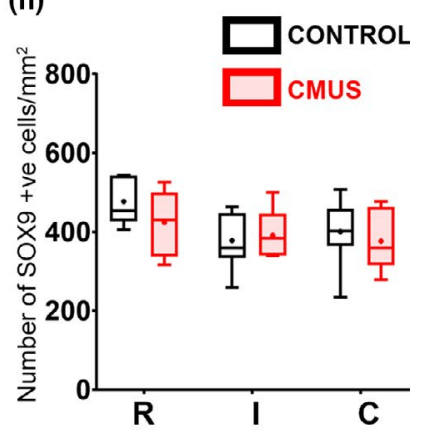

(I)

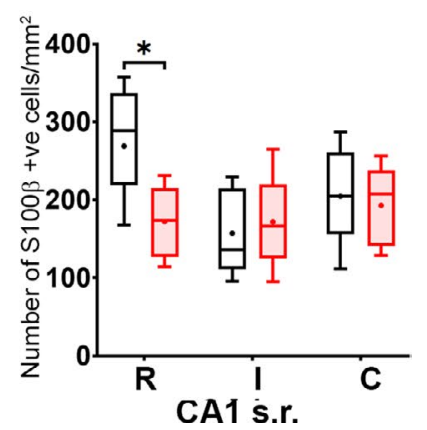

F I G U R E 4 Effects of chronic mild unpredictable stress (CMUS) on astrocyte densities across the rostro-caudal axis in different hippocampal subfields. Mice were subjected to 21 days of CMUS, while the controls were handled similarly without exposure to stress. SOX 9 and S100 $\beta$ immunostained astrocytes were imaged on a confocal microscope. Region of interest (ROI) was marked on 2-D maximum intensity projections encompassing the desired subfield and the number of cells counted was divided by the area of the ROI. The data were represented and analyzed as the number of cells per square millimeter. The data were binned into three categories, namely, Rostral (R), Intermediate (I), and Caudal (C) depending on the bregma levels (see Section 2). We found a statistically significant decrease in the density of SOX9 expressing cells in the caudal molecular layer of the dentate gyrus (e) and the rostral hilus (a,b,f). All other subregions did not reveal statistically significant differences (e-h). We saw a statistically significant decrease in the S100ß-positive cell density in the rostral hippocampus of the hilus (c,d,i) and the stratum radiatum of the CA1 (1). All other comparisons yielded no statistically significant differences (i-1). $n=3-4$ mice per group. Data represented as box and whisker's plot. $* p<0.05$. All comparisons made with mixed-effects model to study the differential effects of dorso-ventral position and stress treatment, followed by Sidak's post hoc test. Scale bars in (a)-(d) are $100 \mu \mathrm{m}$

hoc test) (Figure $4 \mathrm{k}$ ). In the stratum radiatum region of the CA1, we found a significant decrease in the rostral part of CA1 (control: $269 \pm 25.93$, CMUS: $172.2 \pm 19.83)(p=0.03)$, whereas no differences were observed in the intermediate (control: $157.2 \pm 24.66$, CMUS: $171.9 \pm 23.84)(p=0.96)$ and caudal (control: $204.8 \pm 21.57$, CMUS: $192.9 \pm 22.92$ ) $(p=0.97)$ regions (mixed effects analysis to assess interactions between rostro-caudal level and stress treatment, followed by corrected Sidak's post hoc test) (Figure 4l). Together, these results indicate that the $\mathrm{S} 100 \beta$-immunopositive cell density is decreased only in rostral hilus and rostral CA1 as a result of CMUS, indicating region-specific alterations.

\subsection{Effects of CMUS on the morphology of astrocytes in the molecular layer of the DG}

Because we found that CMUS exerts its effects on density of cells expressing SOX9 and S100 $\beta$ mostly in the rostral parts and that intermediate and caudal parts seem to be largely spared in our analysis, we next addressed if CMUS affects the morphology of astrocytes in the rostral sections. The morphological analysis on GFAP-stained images of astrocytes was done as explained in Section 2 and in Figure S3. To assess the effects of CMUS on astrocytic branching on molecular layer astrocytes, we performed Sholl analysis. We 
found a significant decrease in the ramification along most of the radius of cells in CMUS-treated mice as compared to the control mice (two-way repeated measures ANOVA followed by Sidak's post hoc test) (Figure 5a). We then compared the area under the Sholl analysis curve between the controls and CMUS-treated mice and found a significant decrease (control: $85.99 \pm 2.04$, CMUS: $73.12 \pm 1.48)(p=0.006$, nested $t$ test $)$, suggesting a robust decline in ramifications (Figure $5 \mathrm{~b}$ ). We then studied the enclosing radius and found a significant decrease in the CMUS-treated mice as compared to the control mice (control: $27.07 \pm 0.47$, CMUS: $25.02 \pm 0.47)(p=0.04$, nested $t$ test) (Figure 5c). Next, we calculated the total surface area covered by astrocytes from the thresholded maximum intensity projected images and found a significant decrease in the CMUS-treated group as compared to the control mice (control: $214.30 \pm 5.96$, CMUS: $185.40 \pm 4.13)(p=0.0002$, nested $t$ test) (Figure 5d). However, we did not find any difference in the average width of astrocytic processes between the control and CMUS-treated mice (control: $2.35 \pm 0.02$, CMUS: $2.44 \pm 0.03)(p=0.12$, nested $t$ test $)$ (Figure 5e).

\section{5 | Effects of CMUS on the morphology of astrocytes in the hilus}

Next, we assessed the effects of CMUS on astrocytic branching on hilar astrocytes by Sholl analysis. We found a significant decrease in the ramification, but only along a small portion of the proximal segment of astrocytic branches in CMUStreated mice as compared to the control mice (two-way repeated measures ANOVA followed by Sidak's post hoc test) (Figure 6a). However, we found no difference in the area under the Sholl analysis curve between the controls and CMUStreated mice (control: $55.49 \pm 1.49$, CMUS: $51.44 \pm 1.71$ ) ( $p=0.18$, nested $t$ test) (Figure 6b), suggesting only a marginal change in branching at a small portion of proximal (a)

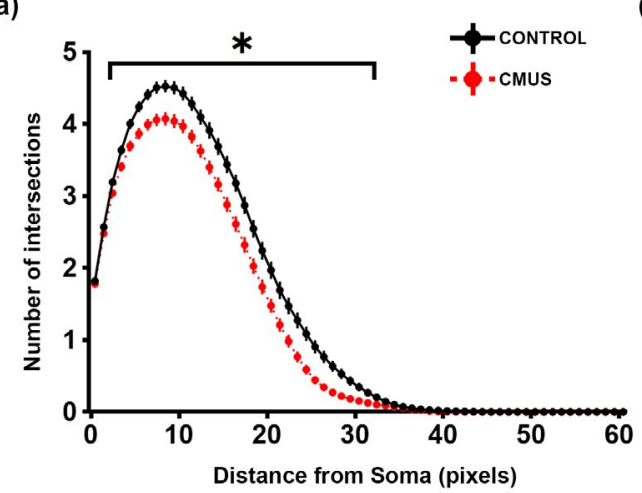

(c)

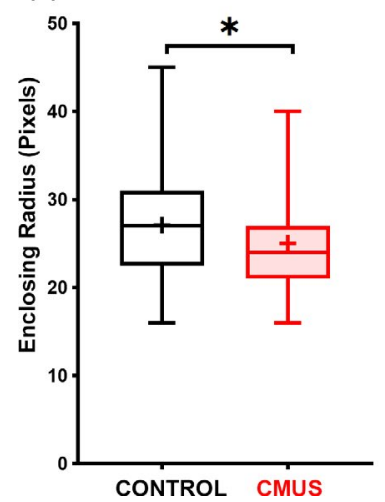

(d)

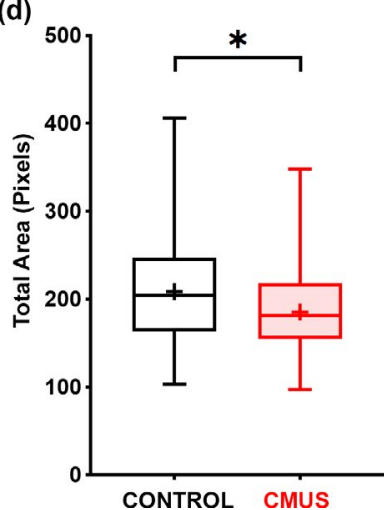

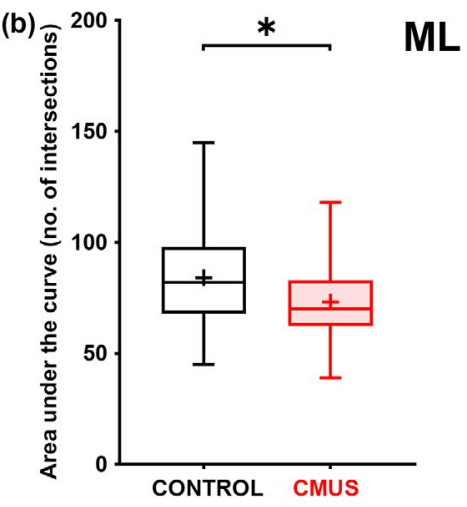

(e)

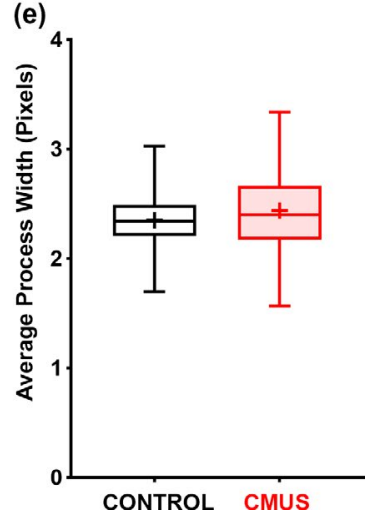

F I G URE 5 Chronic mild unpredictable stress (CMUS) affects the morphology of astrocytes in the molecular layer of the dentate gyrus (DG). Mice were subjected to 21 days of CMUS, while the controls were handled similarly without exposure to stress. GFAP immunostained astrocytes were imaged on a confocal microscope and the images of astrocytes from the molecular layer of the DG (ML) were used for morphological analysis as shown in Figure S3. Line plot depicting Sholl analysis reveals a significant decrease in the ramification of astrocytes in CMUS-treated mice as compared to the control mice (a). Box and whiskers plots show a significant decrease in the area under the Sholl curve (b), enclosing radius (c), and total projected surface area (d) for astrocytes from CMUS-treated mice as compared to the controls. However, no statistically significant difference was seen in the average process width between the two groups (e). $n=$ (control: 145 cells from four mice, CMUS: 121 cells from three mice). Data represented as mean $\pm S E M$ for Sholl analysis and as box and whisker's plot for two group comparisons. For Sholl analysis, $* p<0.05$, two-way repeated measures analysis of variance followed by Sidak's post hoc test. For two group comparisons, ${ }^{*} p<0.05$, nested $t$ test 
(a)

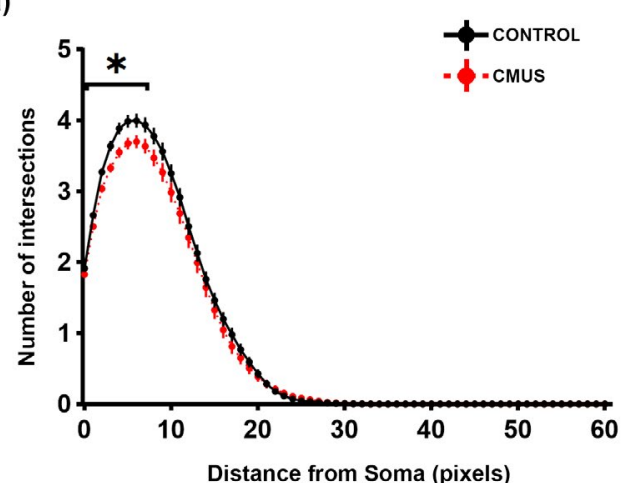

(c)

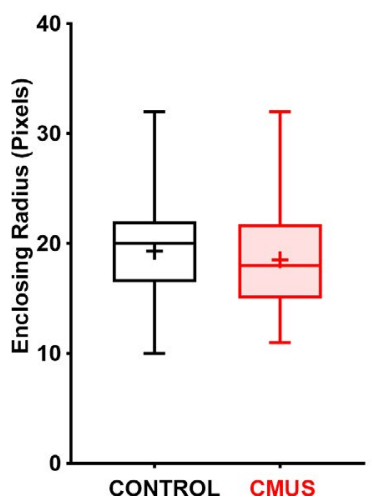

(b)

(d)

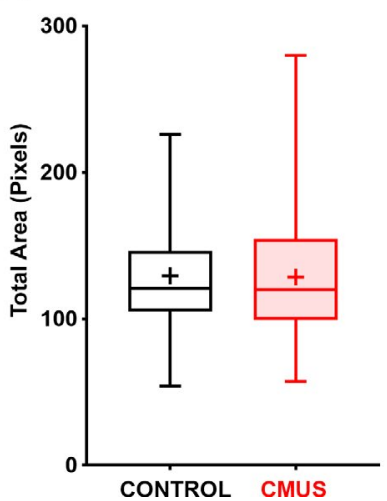

Hilus

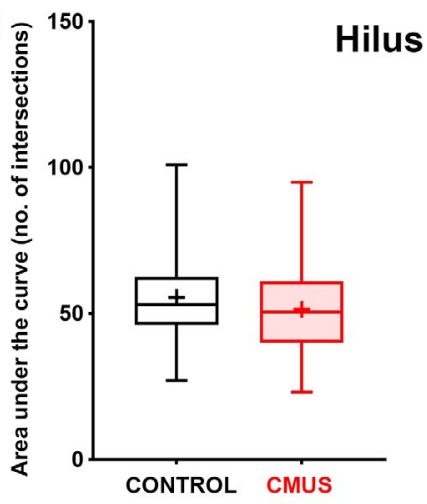

(e)

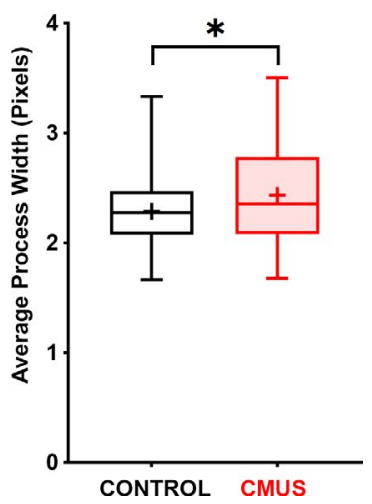

F I G U R E 6 Effects of chronic mild unpredictable stress (CMUS) on the morphology of astrocytes in the hilus. Mice were subjected to 21 days of CMUS, while the controls were handled similarly without exposure to stress. GFAP immunostained astrocytes were imaged on a confocal microscope and the images of astrocytes from the hilar region of the dentate gyrus were analyzed for morphological analysis as shown in Figure S3. Line plot depicting Sholl analysis reveals a significant decrease in the ramification only in the proximal segment of astrocytes in CMUS-treated mice as compared to the control mice (a). Box and whiskers plots show no significant change in the area under the Sholl curve (b), enclosing radius (c), and total projected surface area (d) for astrocytes from CMUS-treated mice as compared to the controls. However, a small (7.56\%) but statistically significant increase was seen in the average process width in the hilar astrocytes of CMUS-treated mice as compared to the control mice (e). $n=$ (control: 109 cells from four mice, CMUS: 84 cells from three mice). Data represented as mean \pm SEM for Sholl analysis and as box and whisker's plot for two group comparisons. For Sholl analysis, ${ }^{*} p<0.05$, two-way repeated measures analysis of variance followed by Sidak's post hoc test. For two group comparisons, ${ }^{*} p<0.05$, nested $t$ test

branches (Figure 6a,b). We also found no statistically significant difference between CMUS-treated mice and control mice in terms of the enclosing radius (control: $19.30 \pm 0.37$, CMUS: $18.52 \pm 0.50)(p=0.42$, nested $t$ test $)$ (Figure 6c) and total surface area (control: $130.50 \pm 3.71$, CMUS: $128.50 \pm 4.77$ ) ( $p=0.95$, nested $t$ test) (Figure 6d). Interestingly, we found a small (7.56\%) but statistically significant ( $p=0.001$, nested $t$ test) increase in average process width in astrocytes of CMUStreated mice as compared to the controls (control: $2.29 \pm 0.03$, CMUS: $2.46 \pm 0.05$ ) (Figure 6e).

\subsection{Effects of CMUS on the morphology of astrocytes in the stratum lucidum of $\mathrm{CA3}$}

In order to study the effects of CMUS on branching of astrocytes from the stratum lucidum of the CA3, Sholl analysis was performed. We found a statistically significant decrease in the ramification but only in a small portion of distal branches of astrocytes in CMUS-treated mice as compared to the control mice (Figure 7a). At the other segments, we did not find any change in ramification (Figure 7a) (two-way repeated measures ANOVA followed by Sidak's post hoc test). We also examined other morphology-related parameters in these astrocytes from the stratum lucidum of CA3. However, we did not find a statistically significant difference in the area under the Sholl curve (control: $85.14 \pm 3.36$, CMUS: $77.92 \pm 2.82)(p=0.21$, nested $t$ test) (Figure 7b), enclosing radius (control: $28.03 \pm 0.96$, CMUS: $27.16 \pm 0.89)(p=0.62$, nested $t$ test) (Figure 7c) or in total area of the cells (control: $214.30 \pm 9.95$, CMUS: $200.50 \pm 9.26)(p=0.46$, nested $t$ test) (Figure 7d) between the control mice and the CMUS-treated mice. Additionally, we did not find a statistically significant difference in the average process width between the $\mathrm{CA} 3$ astrocytes from control mice and the CMUS-treated mice (control: $2.41 \pm 0.04$, CMUS: $2.51 \pm 0.06)(p=0.17$, nested $t$ test $)$ (Figure 7e). 
(a)

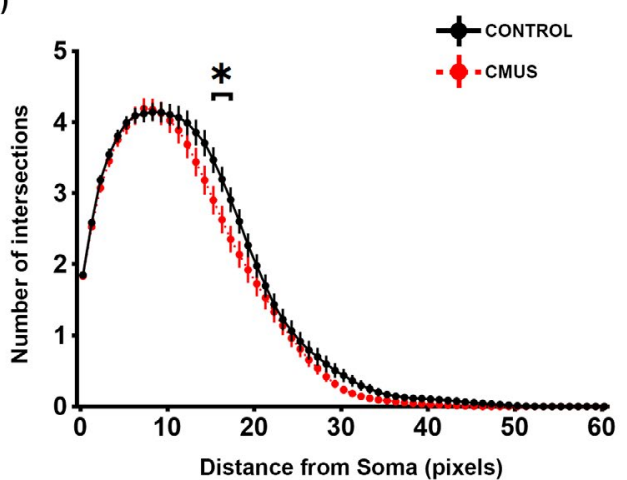

(c)

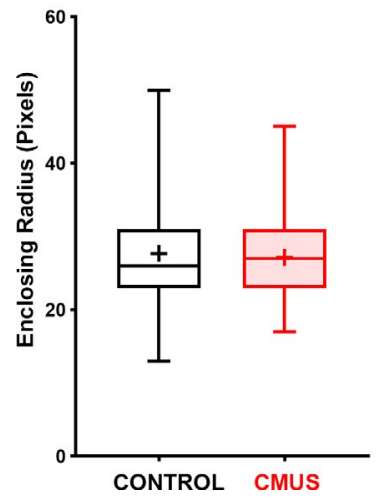

(d)

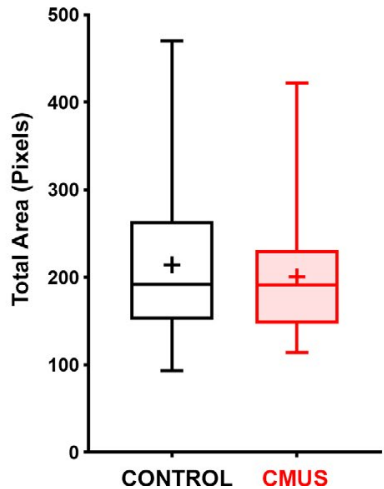

CA3 s.I.

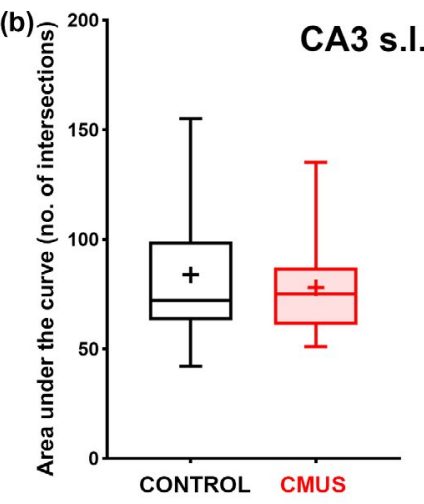

(e)

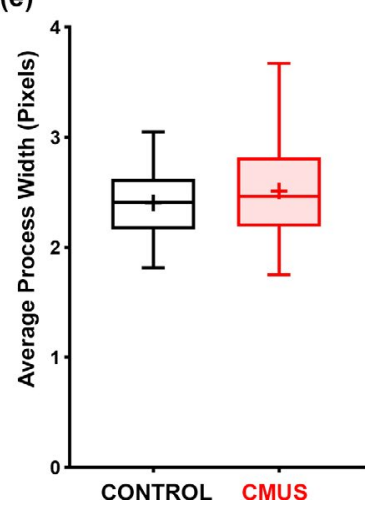

F I G U RE 7 Effects of chronic mild unpredictable stress (CMUS) on the morphology of astrocytes in the stratum lucidum of CA3. Mice were subjected to 21 days of CMUS, while the controls were handled similarly without exposure to stress. GFAP immunostained astrocytes were imaged on a confocal microscope and the images of astrocytes from the stratum lucidum region of the CA3 were analyzed for morphological analysis as shown in Figure S3. Line plot depicting Sholl analysis reveals a significant decrease in the ramification only in a small portion of the distal segment of astrocytes in CMUS-treated mice as compared to the control mice (a). Box and whiskers plots show no significant change in the area under the Sholl curve (B), enclosing radius (c), total projected surface area (d), and average process width (e) for astrocytes from CMUS-treated mice as compared to the controls. $n=$ (control: 72 cells from four mice, CMUS: 51 cells from three mice). Data represented as mean \pm SEM for Sholl analysis and as box and whisker's plot for two group comparisons. For Sholl analysis, ${ }^{*} p<0.05$, two-way repeated measures analysis of variance followed by Sidak's post hoc test. Two group comparisons were analyzed with nested $t$ test

\section{7 | Effects of CMUS on the morphology of astrocytes in the stratum radiatum of CA1}

We further investigated the effects of CMUS on astrocytic branching in the stratum radiatum of the CA1 using Sholl analysis. However, we did not find any difference in ramification between astrocytes from CMUS-treated mice as compared to the control mice (two-way repeated measures ANOVA followed by Sidak's post hoc test) (Figure 8a). Furthermore, we did not find a statistically significant difference in the area under the Sholl curve (control: $81.74 \pm 2.59$, CMUS: $73.74 \pm 2.62)(p=0.25$, nested $t$ test) (Figure $8 b$ ), enclosing radius (control: $28.19 \pm 0.81$, CMUS: $26.27 \pm 0.89)(p=0.18$, nested $t$ test) (Figure 8c) or in total area of the cells (control: $197.40 \pm 7.47$, CMUS: $177.50 \pm 8.30)(p=0.30$, nested $t$ test) (Figure 8d). We also did not find any difference in the average process width between the CA1 stratum radiatum astrocytes from control mice and the CMUS-treated mice (control: $2.33 \pm 0.03$, CMUS: $2.31 \pm 0.05)(p=0.76$, nested $t$ test $)$ (Figure 8e).
These results indicate that the astrocytes from CA1 stratum radiatum are not affected morphologically in our CMUS paradigm.

\section{DISCUSSION}

Hippocampus is an integral part of the limbic system and is implicated in the pathophysiology of MDD as well as in the action of antidepressant drugs (Nestler et al., 2002). Moreover, astrocytic populations in specific subfields of the hippocampus seem to be differentially vulnerable to the pathology associated with MDD (Cobb et al., 2013, 2016). The quest for the mechanistic understanding of these differential vulnerabilities between different astrocytic subpopulations of the hippocampus necessitates a careful profiling in a tractable model system such as mice. In this study, we used CMUS mouse model and assessed its effects on the cell densities and morphology of hippocampal astrocytes from different subfields (Table 1). We found that the cell 
(a)

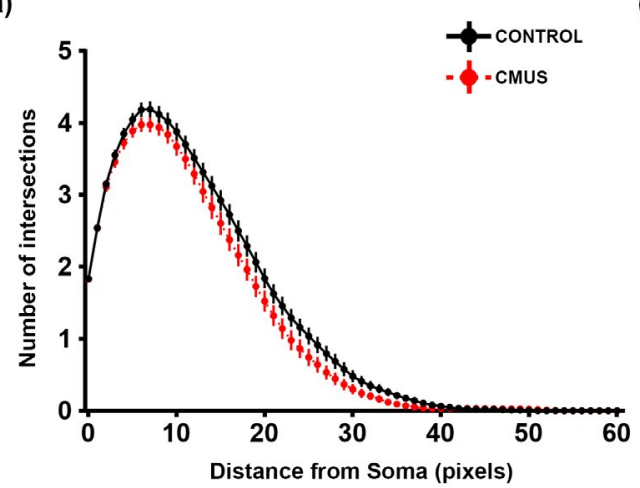

(c)

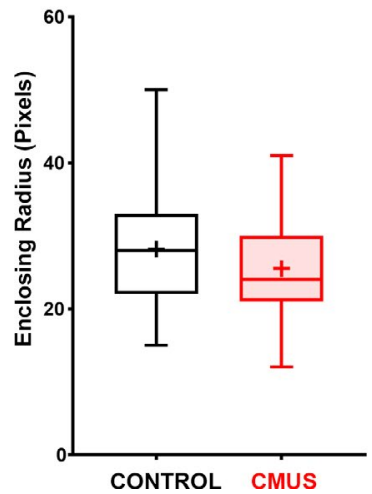

(b)

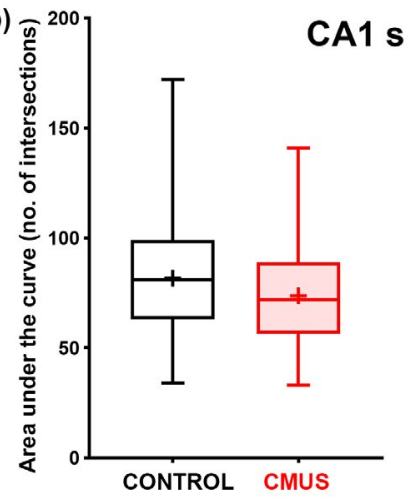

(e) (d)

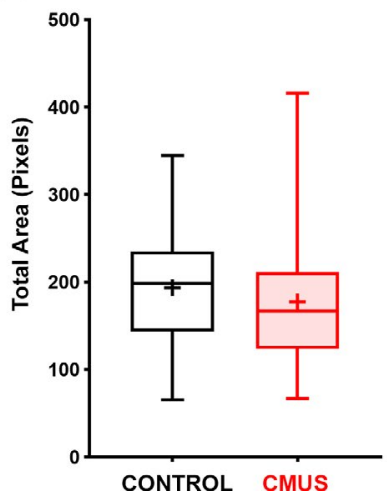

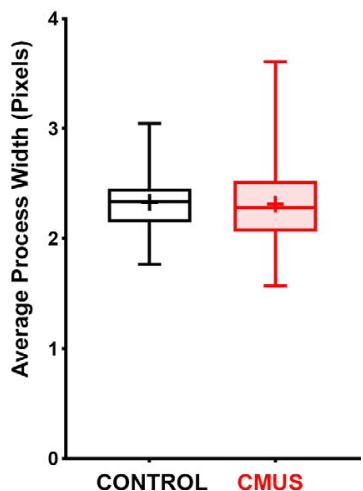

F I G U R E 8 Effects of chronic mild unpredictable stress (CMUS) on the morphology of astrocytes in the stratum radiatum of CA1. Mice were subjected to 21 days of CMUS, while the controls were handled similarly without exposure to stress. GFAP immunostained astrocytes were imaged on a confocal microscope and the images of astrocytes from the stratum radiatum region of the CA1 were analyzed for morphological analysis as shown in Figure S3. Line plot depicting Sholl analysis reveals no significant difference in the ramification of astrocytes in CMUStreated mice as compared to the control mice (a). Additionally, box and whiskers plots show no significant change in the area under the Sholl curve (b), enclosing radius (c), total projected surface area (d), and average process width (e) for astrocytes from CMUS-treated mice as compared to the controls. $n=$ (control: 91 cells from four mice, CMUS: 73 cells from three mice). Data represented as mean \pm SEM for Sholl analysis and as box and whisker's plot for two group comparisons. Sholl analysis data were analyzed by two-way repeated measures analysis of variance followed by Sidak's post hoc test. Two group comparisons were analyzed with nested $t$ test

TA B LE 1 Summary of the effects of CMUS on astrocyte morphology

\begin{tabular}{|c|c|c|c|c|c|}
\hline & $\begin{array}{l}\text { Sholl analysis (ramification of } \\
\text { astrocytes) }\end{array}$ & $\begin{array}{l}\text { Area under the } \\
\text { Sholl curve }\end{array}$ & $\begin{array}{l}\text { Enclosing } \\
\text { radius (pixels) }\end{array}$ & $\begin{array}{l}\text { Total area of } \\
\text { cells (pixels) }\end{array}$ & $\begin{array}{l}\text { Average process } \\
\text { width (pixels) }\end{array}$ \\
\hline Molecular layer & $\begin{array}{l}\text { Strong decline in astrocytic } \\
\text { process ramifications }\end{array}$ & Decreased & Decreased & Decreased & No change \\
\hline Hilus & $\begin{array}{l}\text { Small decrease in astrocytic } \\
\text { process ramifications }\end{array}$ & No change & No change & No change & Marginally increased \\
\hline CA1 stratum radiatum & Process ramifications not affected & No change & No change & No change & No change \\
\hline
\end{tabular}

Note: The table shows the summary of the effects of CMUS on the morphology of hippocampal astrocytes from molecular layer of the DG, Hilus, CA3 stratum lucidum, and CA1 stratum radiatum.

density was significantly lowered in selective hippocampal subfields, with the rostral part being especially vulnerable. Furthermore, in terms of morphological characteristics, molecular layer was found to be the most affected subfield, followed by the hilus. CA3 astrocytes were much less affected, while the astrocytes in CA1 were largely spared. These results corroborate the observations in MDD patients showing that the astrocytic subpopulations from different hippocampal subfields are differentially vulnerable to MDD pathology (Cobb et al., 2013, 2016). 


\section{1 | Analysis of astrocyte morphology in animal models of stress}

Studies in animal models have shown divergent results regarding the effects of stress on astrocytes. It was shown that chronic psychosocial stress in treeshrews decreases the number and somal volume of hippocampal astrocytes, which was reversed by chronic fluoxetine treatment (Czéh et al., 2005). On the other hand, studies have also shown no differences in the hippocampal astrocytes between stressed groups and controls (Musholt et al., 2009; Naskar \& Chattarji, 2019). Another study also showed an increase in the hippocampal GFAP immunoreactivity after chronic immobilization stress (Jang et al., 2008). However, these differences can arise from differences in experimental stress paradigms and the subfields of the hippocampus that were studied. Consistent with some of these studies, we found no differences in astrocyte morphology in the dorsal CA3 (Naskar \& Chattarji, 2019) (Figure 7), as well as no statistically significant differences in the number of astrocytes in CA1, when the sections were taken spanning the rostro-caudal extent (Musholt et al., 2009) (Figure 3d,h). Our study addresses some of these concerns by simultaneously analyzing different hippocampal subfields in the same group of mice. We believe that these results can be useful to further probe the mechanisms underlying differential vulnerabilities to stress between different astrocytic subpopulations.

These studies also raise further questions regarding the effects of stress on astrocytic structure and function. Fine leaflet-like process, also called perisynaptic astrocytic process, ensheath synapses in the central nervous system and play an integral role in the regulation of synaptic transmission (Bernardinelli et al., 2014). However, GFAP only stains about $15 \%$ of the GFAP somal volume revealing only the core astrocytic processes. Hence, future studies will need to address the plasticity at the perisynaptic astrocytic processes.

Such studies usually require dye-filling experiments to stain the cytoplasm in its entirety (Medvedev et al., 2014; Naskar \& Chattarji, 2019). This makes these experiments time consuming, while severely limiting the ability to analyze large sample sizes across different brain regions. Sparsely labeling the astrocytes in vivo with genetically encoded membrane-targeted fluorescent proteins would allow the analysis of large datasets encompassing various brain regions across several experimental paradigms. Automation of some of the steps in the image-processing pipeline would further facilitate these large-scale analyses.

\section{2 | Differential involvement of dorsal versus ventral hippocampus in pathophysiology of depression}

Hippocampus is a large brain structure neatly arranged into a trisynaptic circuitry, which is involved in learning and memory as well as emotional behavior. Interestingly, hippocampus exhibits neuroanatomical (Ruth et al., 1982; Van Groen \& Lopes da Silva, 1985; Witter, 1986), molecular (Cembrowski, Bachman, et al., 2016; Cembrowski, Wang, et al., 2016; Lee et al., 2017), electrophysiological (Witter, 1986), and functional (Bannerman et al., 2003; Moser \& Moser, 1998) divergence along its posterio-anterior axis, which corresponds to the dorso-ventral axis in rodents. While the posterior hippocampus, or dorsal hippocampus in rodents is involved in memory encoding (Moser \& Moser, 1998), the anterior or ventral hippocampus is involved in processing emotional information (Bannerman et al., 2003). Supporting this view of functional segregation, it was shown that astrocyte cell density in the anterior hippocampus was decreased in depressed female monkeys (Willard et al., 2013). In this study, only the astrocyte density in CA1 and DG was selectively diminished (Willard et al., 2013). Intriguingly, we found that the SOX 9 and S100 $\beta$-immunopositive cell density is lower in stressed mice in the rostral (Bregma -1.46 to -1.94 ) coronal sections in selective subfields, which correspond to the dorsal hippocampus, while we saw that in the sections from more caudal (ventral) hippocampus, cell density remained unchanged, except for SOX9-expressing cells in the molecular layer. This is indeed interesting, given that ventral hippocampus receives denser innervations from the hypothalamus, the master regulator of stress responses. That said, the caudal coronal sections (Bregma -2.54 to -2.92 ) from rodent brain are not entirely ventral hippocampal but have sizable portions from the intermediate hippocampus. Transverse sections of dissected hippocampi allow a bonafide distinction between the dorsal and ventral poles of the hippocampus. Further studies are needed to ascertain whether astrocyte density in the ventral hippocampus is indeed unaltered in CMUS-treated mice.

\section{3 | Astrocytic atrophy as a potential pathogenic event}

Psychiatric and neurodegenerative disorders can be viewed as synaptopathies (Brose et al., 2010). Astrocytic atrophy and dysfunction has been reported in several neurological and psychiatric disorders, including MDD (Cotter et al., 2001; Miguel-Hidalgo et al., 2000; Ongür et al., 1998; Rajkowska et al., 1999; Rajkowska \& Stockmeier, 2013; Verkhratsky et al., 2014). Atrophy in astrocytes leads to reduction in synaptic coverage thereby compromising the wellbeing of synapses (Verkhratsky et al., 2016). This phenomenon may manifest itself through reduced glutamate uptake, diminished trophic support, or disruption of metabolic coupling (Elsayed \& Magistretti, 2015; Marathe et al., 2018; Martin et al., 2013). Despite such a consequential nature of astroglial atrophy in several brain disorders, the molecular mechanisms underlying the atrophy remain elusive. It was shown 
that selective ablation of prefrontal astrocytes in rats was sufficient to induce depressive-like symptoms in experimental rats (Banasr \& Duman, 2008). Hence, it is conceivable that preventing the glial degeneration associated with MDD may prove to be a viable therapeutic strategy. The changes observed in this study in GFAP profiles may either signify astroglial atrophy or may just be a result of stress-induced structural plasticity. Further mechanistic studies are needed to decipher the significance of the changes in GFAP profiles in stressed mice.

\section{CONCLUSIONS}

In conclusion, while majority of MDD research so far has focused on neurons, the astrocytic degeneration and dysfunction has remained relatively unexplored. The data from this manuscript highlight the fact that not all astrocytes within the hippocampus are uniformly affected by stress. This corroborates previous studies involving postmortem analyses in the victims of MDD showing differential vulnerabilities within hippocampal subfields (Cobb et al., 2013, 2016). Given the integral role of hippocampus in MDD pathophysiology and in antidepressant action, astrocytic degeneration in selective subfields of hippocampus may have important functional ramifications.

\section{ACKNOWLEDGMENTS}

This work was supported by INSPIRE faculty grant from Department of Science and Technology (DST), India (DST/ INSPIRE/04-I/2016/000002) to SM, and Early Career Research Award from Scientific and Engineering Research Board (SERB) to SM (ECR/2017/003240). GV and AN were supported by CSIR-NET Junior and Senior Research Fellowship. Authors are grateful to Prof. Naren Ramanan for S100 $\beta$ antibody and critical comments on this manuscript. The authors would also like to thank Mr. Manjunath and the staff at central animal facility, IISc and bioimaging facility, IISc.

\section{CONFLICT OF INTEREST}

The authors declare that the research was conducted in the absence of any commercial or financial relationships that could be construed as a potential conflict of interest.

\section{AUTHOR CONTRIBUTIONS}

SM conceived and designed the study. PD, GV, and SM performed experiments. GV analyzed the data. AN performed FST and OFT behavior analysis. SM wrote the manuscript. All authors discussed, proofread, and approved the final version.

\section{PEER REVIEW}

The peer review history for this article is available at https:// publons.com/publon/10.1111/ejn.15234.

\section{DATA AVAILABILITY STATEMENT}

Requests for raw data can be addressed to the corresponding author, and the data will be made available upon reasonable request.

\section{ORCID}

Swananda Marathe (iD https://orcid.

org/0000-0002-2539-366X

\section{REFERENCES}

Banasr, M., \& Duman, R. S. (2008). Glial loss in the prefrontal cortex is sufficient to induce depressive-like behaviors. Biological Psychiatry, 64, 863-870. https://doi.org/10.1016/j.biopsych.2008.06.008

Bannerman, D. M., Grubb, M., Deacon, R. M. J., Yee, B. K., Feldon, J., \& Rawlins, J. N. P. (2003). Ventral hippocampal lesions affect anxiety but not spatial learning. Behavioral Brain Research, 139, 197-213. https://doi.org/10.1016/S0166-4328(02)00268-1

Bernardinelli, Y., Muller, D., \& Nikonenko, I. (2014). Astrocytesynapse structural plasticity. Neural Plasticity, 2014, 1-13. https:// doi.org/10.1155/2014/232105

Bremner, J. D., Vythilingam, M., Vermetten, E., Nazeer, A., Adil, J., Khan, S., Staib, L. H., \& Charney, D. S. (2002). Reduced volume of orbitofrontal cortex in major depression. Biological Psychiatry, 51, 273-279. https://doi.org/10.1016/S0006-3223(01)01336-1

Brose, N., O'Connor, V., \& Skehel, P. (2010). Synaptopathy: Dysfunction of synaptic function? Biochemical Society Transactions, 38, 443-444

Brown, E. S., Hughes, C. W., McColl, R., Peshock, R., King, K. S., \& Rush, A. J. (2014). Association of depressive symptoms with hippocampal volume in 1936 adults. Neuropsychopharmacology, 39, 770-779. https://doi.org/10.1038/npp.2013.271

Castrén, E., \& Hen, R. (2013). Neuronal plasticity and antidepressant actions. Trends in Neurosciences, 36, 259-267. https://doi. org/10.1016/j.tins.2012.12.010

Cembrowski, M. S., Bachman, J. L., Wang, L., Sugino, K., Shields, B. C., \& Spruston, N. (2016). Spatial gene-expression gradients underlie prominent heterogeneity of CA1 Pyramidal Neurons. Neuron, 89, 351-368. https://doi.org/10.1016/j.neuron.2015.12.013

Cembrowski, M. S., Wang, L., Sugino, K., Shields, B. C., \& Spruston, N. (2016). Hipposeq: A comprehensive RNA-seq database of gene expression in hippocampal principal neurons. Elife, 5, e14997. https://doi.org/10.7554/eLife.14997

Cobb, J. A., O’Neill, K., Milner, J., Mahajan, G. J., Lawrence, T. J., May, W. L., Miguel-Hidalgo, J., Rajkowska, G., \& Stockmeier, C. A. (2016). Density of GFAP-immunoreactive astrocytes is decreased in left hippocampi in major depressive disorder. Neuroscience, 316, 209-220. https://doi.org/10.1016/j.neuroscience.2015.12.044

Cobb, J. A., Simpson, J., Mahajan, G. J., Overholser, J. C., Jurjus, G. J., Dieter, L., Herbst, N., May, W., Rajkowska, G., \& Stockmeier, C. A. (2013). Hippocampal volume and total cell numbers in major depressive disorder. Journal of Psychiatric Research, 47, 299-306. https://doi.org/10.1016/j.jpsychires.2012.10.020

Cotter, D., Mackay, D., Landau, S., Kerwin, R., \& Everall, I. (2001). Reduced glial cell density and neuronal size in the anterior cingulate cortex in major depressive disorder. Archives of General Psychiatry, 58, 545-553. https://doi.org/10.1001/archpsyc.58.6.545

Czéh, B., Simon, M., Schmelting, B., Hiemke, C., \& Fuchs, E. (2005). Astroglial plasticity in the hippocampus is affected by 
chronic psychosocial stress and concomitant fluoxetine treatment. Neuropsychopharmacology, 31, 1616-1626. https://doi. org/10.1038/sj.npp.1300982

Dai, L., Zhou, H., Xu, X., \& Zuo, Z. (2019). Brain structural and functional changes in patients with major depressive disorder: A literature review. PeerJ, 7. https://doi.org/10.7717/peerj.8170

Drevets, W. C. (2000). Functional anatomical abnormalities in limbic and prefrontal cortical structures in major depression. Progress in Brain Research, 126, 413-431.

Duman, C. H., \& Duman, R. S. (2015). Spine synapse remodeling in the pathophysiology and treatment of depression. Neuroscience Letters, 601, 20-29. https://doi.org/10.1016/j.neulet.2015.01.022

Elsayed, M., \& Magistretti, P. J. (2015). A new outlook on mental illnesses: Glial involvement beyond the glue. Frontiers in Cellular Neuroscience, 9, 468. https://doi.org/10.3389/fncel.2015.00468

Grønli, J., Murison, R., Fiske, E., Bjorvatn, B., Sørensen, E., Portas, C. M., \& Ursin, R. (2005). Effects of chronic mild stress on sexual behavior, locomotor activity and consumption of sucrose and saccharine solutions. Physiology \& Behavior, 84, 571-577. https://doi. org/10.1016/j.physbeh.2005.02.007

Jang, S., Suh, S. H., Yoo, H.-S., Lee, Y.-M., \& Oh, S. (2008). Changes in iNOS, GFAP and NR1 expression in various brain regions and elevation of sphingosine-1-phosphate in serum after immobilized stress. Neurochemical Research, 33, 842-851. https://doi. org/10.1007/s11064-007-9523-6

Kempton, M. J., Salvador, Z., Munafò, M. R., Geddes, J. R., Simmons, A., Frangou, S., \& Williams, S. C. R. (2011). Structural neuroimaging studies in major depressive disorder. Meta-analysis and comparison with bipolar disorder. Archives of General Psychiatry, 68, 675-690. https://doi.org/10.1001/archgenpsychiatry.2011.60

Lee, A.-R., Kim, J.-H., Cho, E., Kim, M., \& Park, M. (2017). Dorsal and ventral hippocampus differentiate in functional pathways and differentially associate with neurological disease-related genes during postnatal development. Frontiers in Molecular Neuroscience, 10. https://doi.org/10.3389/fnmol.2017.00331

Lorenzetti, V., Allen, N. B., Fornito, A., \& Yücel, M. (2009). Structural brain abnormalities in major depressive disorder: A selective review of recent MRI studies. Journal of Affective Disorders, 117, 1-17. https://doi.org/10.1016/j.jad.2008.11.021

Marathe, S. V., D'almeida, P. L., Virmani, G., Bathini, P., \& Alberi, L. (2018). Effects of monoamines and antidepressants on astrocyte physiology: Implications for monoamine hypothesis of depression. Journal of Experimental Neuroscience, 12, 1179069518789149. https://doi.org/10.1177/1179069518789149

Marathe, S., Liu, S., Brai, E., Kaczarowski, M., \& Alberi, L. (2015). Notch signaling in response to excitotoxicity induces neurodegeneration via erroneous cell cycle reentry. Cell Death and Differentiation. https://doi.org/10.1038/cdd.2015.23

Martin, J.-L., Magistretti, P. J., \& Allaman, I. (2013). Regulation of neurotrophic factors and energy metabolism by antidepressants in astrocytes. Current Drug Targets, 14, 1308-1321.

McKinnon, M. C., Yucel, K., Nazarov, A., \& MacQueen, G. M. (2009). A meta-analysis examining clinical predictors of hippocampal volume in patients with major depressive disorder. Journal of Psychiatry and Neuroscience, 34, 41-54.

Medvedev, N., Popov, V., Henneberger, C., Kraev, I., Rusakov, D. A., \& Stewart, M. G. (2014). Glia selectively approach synapses on thin dendritic spines. Philosophical Transactions of the Royal Society of London. Series B, Biological Sciences, 369. https://doi.org/10.1098/ rstb.2014.0047
Miguel-Hidalgo, J. J., Baucom, C., Dilley, G., Overholser, J. C., Meltzer, H. Y., Stockmeier, C. A., \& Rajkowska, G. (2000). Glial fibrillary acidic protein immunoreactivity in the prefrontal cortex distinguishes younger from older adults in major depressive disorder. Biological Psychiatry, 48, 861-873. https://doi.org/10.1016/ S0006-3223(00)00999-9

Miguel-Hidalgo, J. J., Waltzer, R., Whittom, A. A., Austin, M. C., Rajkowska, G., \& Stockmeier, C. A. (2010). Glial and glutamatergic markers in depression, alcoholism, and their comorbidity. Journal of Affective Disorders, 127, 230-240. https://doi.org/10.1016/j. jad.2010.06.003

Moser, M. B., \& Moser, E. I. (1998). Functional differentiation in the hippocampus. Hippocampus, 8, 608-619. https://doi.org/10.1002/ (SICI) 1098-1063(1998)8:6<608:AID-HIPO3>3.0.CO;2-7

Musholt, K., Cirillo, G., Cavaliere, C., Bianco, M. R., Bock, J., Helmeke, C., Braun, K., \& Papa, M. (2009). Neonatal separation stress reduces glial fibrillary acidic protein- and S100 $\beta$-immunoreactive astrocytes in the rat medial precentral cortex. Developmental Neurobiology, 69, 203-211. https://doi.org/10.1002/dneu.20694

Naskar, S., \& Chattarji, S. (2019). Stress elicits contrasting effects on the structure and number of astrocytes in the amygdala versus hippocampus. Eneuro, 6. https://doi.org/10.1523/ENEURO.0338-18.2019

Nestler, E. J., Barrot, M., DiLeone, R. J., Eisch, A. J., Gold, S. J., \& Monteggia, L. M. (2002). Neurobiology of depression. Neuron, 34, 13-25. https://doi.org/10.1016/S0896-6273(02)00653-0

Ongür, D., Drevets, W. C., \& Price, J. L. (1998). Glial reduction in the subgenual prefrontal cortex in mood disorders. Proceedings of the National Academy of Sciences of the United States of America, 95, 13290-13295. https://doi.org/10.1073/pnas.95.22.13290

Qiao, H., Li, M.-X., Xu, C., Chen, H.-B., An, S.-C., \& Ma, X.M. (2016). Dendritic spines in depression: What we learned from animal models. Neural Plasticity, 2016, 1-26. https://doi. org/10.1155/2016/8056370

Rajkowska, G., Miguel-Hidalgo, J. J., Wei, J., Dilley, G., Pittman, S. D., Meltzer, H. Y., Overholser, J. C., Roth, B. L., \& Stockmeier, C. A. (1999). Morphometric evidence for neuronal and glial prefrontal cell pathology in major depression. Biological Psychiatry, 45, 1085-1098.

Rajkowska, G., \& Stockmeier, C. A. (2013). Astrocyte pathology in major depressive disorder: Insights from human postmortem brain tissue. Current Drug Targets, 14, 1225-1236.

Ruth, R. E., Collier, T. J., \& Routtenberg, A. (1982). Topography between the entorhinal cortex and the dentate septotemporal axis in rats: I. Medial and intermediate entorhinal projecting cells. The Journal of Comparative Neurology, 209, 69-78. https://doi. org/10.1002/cne.902090107

Santarelli, L., Saxe, M., Gross, C., Surget, A., Battaglia, F., Dulawa, S., Weisstaub, N., Lee, J., Duman, R., Arancio, O., \& Belzung, C. (2003). Requirement of hippocampal neurogenesis for the behavioral effects of antidepressants. Science, 301, 805-809. https://doi. org/10.1126/science. 1083328

Sequeira-Cordero, A., Salas-Bastos, A., Fornaguera, J., \& Brenes, J. C. (2019). Behavioural characterisation of chronic unpredictable stress based on ethologically relevant paradigms in rats. Scientific Reports, 9, 17403. https://doi.org/10.1038/s41598-019-53624-1

Sheline, Y. I. (2003). Neuroimaging studies of mood disorder effects on the brain. Biological Psychiatry, 54, 338-352. https://doi. org/10.1016/S0006-3223(03)00347-0

Sheline, Y. I., Wang, P. W., Gado, M. H., Csernansky, J. G., \& Vannier, M. W. (1996). Hippocampal atrophy in recurrent major depression. 
Proceedings of the National Academy of Sciences of the United States of America, 93, 3908-3913. https://doi.org/10.1073/ pnas.93.9.3908

Sousa, N., Lukoyanov, N. V., Madeira, M. D., Almeida, O. F. X., \& Paula-Barbosa, M. M. (2000). Reorganization of the morphology of hippocampal neurites and synapses after stress-induced damage correlates with behavioral improvement. Neuroscience, 97, 253-266. https://doi.org/10.1016/S0306-4522(00)00050-6

Sun, W., Cornwell, A., Li, J., Peng, S., Osorio, M. J., Aalling, N., Wang, S. U., Benraiss, A., Lou, N., Goldman, S. A., \& Nedergaard, M. (2017). SOX9 is an astrocyte-specific nuclear marker in the adult brain outside the neurogenic regions. Journal of Neuroscience, 37, 4493-4507. https://doi.org/10.1523/JNEUROSCI.3199-16.2017

Van Groen, T., \& Lopes da Silva, F. H. (1985). Septotemporal distribution of entorhinal projections to the hippocampus in the cat: Electrophysiological evidence. The Journal of Comparative Neurology, 238, 1-9. https://doi.org/10.1002/cne.902380102

Verkhratsky, A., Rodríguez, J. J., \& Steardo, L. (2014). Astrogliopathology: A central element of neuropsychiatric diseases? Neuroscientist, 20, 576-588. https://doi.org/10.1177/1073858413 510208

Verkhratsky, A., Steardo, L., Parpura, V., \& Montana, V. (2016). Translational potential of astrocytes in brain disorders. Progress in Neurobiology, 144, 188-205. https://doi.org/10.1016/j.pneur obio.2015.09.003

Willard, S. L., Riddle, D. R., Forbes, M. E., \& Shively, C. A. (2013). Cell number and neuropil alterations in subregions of the anterior hippocampus in a female monkey model of depression. Biological Psychiatry, 74. https://doi.org/10.1016/j.biopsych.2013.03.013
Wilson, M. D., Sethi, S., Lein, P. J., \& Keil, K. P. (2017). Valid statistical approaches for analyzing sholl data: Mixed effects versus simple linear models. Journal of Neuroscience Methods, 279, 33-43. https:// doi.org/10.1016/j.jneumeth.2017.01.003

Witter, M. P. (1986). A survey of the anatomy of the hippocampal formation, with emphasis on the septotemporal organization of its intrinsic and extrinsic connections. Advances in Experimental Medicine and Biology, 203, 67-82.

Yanpallewar, S. U., Fernandes, K., Marathe, S. V., Vadodaria, K. C., Jhaveri, D., Rommelfanger, K., Ladiwala, U., Jha, S., Muthig, V., Hein, L., Bartlett, P., Weinshenker, D., \& Vaidya, V. A. (2010). $\alpha 2-$ adrenoceptor blockade accelerates the neurogenic, neurotrophic, and behavioral effects of chronic antidepressant treatment. Journal of Neuroscience, 30, 1096-1109. https://doi.org/10.1523/JNEUR OSCI.2309-09.2010

\section{SUPPORTING INFORMATION}

Additional supporting information may be found online in the Supporting Information section.

How to cite this article: Virmani G, D'almeida P, Nandi A, Marathe S. Subfield-specific effects of chronic mild unpredictable stress on hippocampal astrocytes. Eur J Neurosci. 2021;00:1-17. https://doi. org/10.1111/ejn.15234 